\title{
Effects of Housing Condition and Acute Nicotine on Impulsive Choice in Lewis and Fischer 344 Rats
}

Jenny E. Ozga

Follow this and additional works at: https://researchrepository.wvu.edu/etd

\section{Recommended Citation}

Ozga, Jenny E., "Effects of Housing Condition and Acute Nicotine on Impulsive Choice in Lewis and Fischer 344 Rats" (2016). Graduate Theses, Dissertations, and Problem Reports. 6365.

https://researchrepository.wvu.edu/etd/6365

This Thesis is protected by copyright and/or related rights. It has been brought to you by the The Research Repository @ WVU with permission from the rights-holder(s). You are free to use this Thesis in any way that is permitted by the copyright and related rights legislation that applies to your use. For other uses you must obtain permission from the rights-holder(s) directly, unless additional rights are indicated by a Creative Commons license in the record and/ or on the work itself. This Thesis has been accepted for inclusion in WVU Graduate Theses, Dissertations, and Problem Reports collection by an authorized administrator of The Research Repository @ WVU. For more information, please contact researchrepository@mail.wvu.edu. 
Effects of Housing Condition and Acute Nicotine on Impulsive Choice in Lewis and Fischer 344 Rats

\author{
Jenny E. Ozga
}

Thesis submitted to the Eberly College of Arts and Sciences at West Virginia University

in partial fulfillment of the requirements for the degree of

Master of Science in Psychology

Committee Members:

Karen G. Anderson, Ph.D., Chair

Elizabeth G.E. Kyonka, Ph.D.

Melissa D. Blank, Ph.D.

Department of Psychology

Morgantown, West Virginia 2016

Keywords: delay discounting, impulsive choice, environmental enrichment, nicotine, rat

Copyright 2016 Jenny E. Ozga 


\begin{abstract}
Effects of Housing Condition and Acute Nicotine on Impulsive Choice in

Lewis and Fischer 344 Rats
\end{abstract}

\title{
Jenny E. Ozga
}

Impulsive choice is defined as choice for a smaller, more immediate reinforcer relative to a larger, delayed reinforcer, and is commonly studied using delay-discounting procedures. There are many variables that contribute to impulsive choice, including biological and environmental determinants. The present study examined both of these variables by evaluating effects of housing condition (single versus paired) on impulsive choice in Lewis (LEW) and Fischer 344 (F344) rats, at baseline and following acute nicotine administration. A within-session procedure was used in which choice was between one food pellet delivered immediately and three food pellets delivered after a delay. The delay to three food pellets was increased systematically across blocks within a session and indifference points were calculated. Paired housing significantly reduced impulsive choice for both rat strains relative to single housing (archival data). In addition, when singly housed, choice was more impulsive for LEW rats relative to F344 rats at baseline. When pair housed, this strain difference was attenuated. Following acute nicotine administration, impulsive choice was significantly reduced at $0.3 \mathrm{mg} / \mathrm{kg}$ for singly housed LEW rats and $1.0 \mathrm{mg} / \mathrm{kg}$ for singly housed F344 rats. However, when pair housed, 0.3 and $1.0 \mathrm{mg} / \mathrm{kg}$ both reduced impulsive choice regardless of rat strain. Thus, previously reported effects of environmental enrichment (i.e., reduced impulsive choice) seem to be, as least in part, due to social housing. It also appears as though effects of nicotine on impulsive choice are baseline-dependent and paired housing may make impulsive choice in F344 rats more sensitive to effects of nicotine. 


\section{ACKNOWLEDGEMENTS}

I would like to thank Dr. Karen G. Anderson, Dr. Melissa D. Blank, and Dr. Elizabeth G.E. Kyonka for serving on my thesis committee and for offering valuable feedback throughout this project. I would like to specifically thank my advisor and mentor, Dr. Karen G. Anderson for her everlasting support, encouragement, and guidance throughout this project and my graduate career. In addition, I thank Dr. Melissa D. Blank for sharing her knowledge of statistics with me. Thank you to my lab-mate, Marissa Turturici, for nonstop teaching and guidance with laboratory procedures and graphical techniques, and to Alex Castillo, Elizabeth Janeiro, and Tyler Nighbor for assisting with data collection. Thank you to Nicholas Felicione for always asking the tough questions and making me think critically. A very special thank you to my parents, John and Mary Ozga, and siblings, Joseph Ozga and Lindsay Mahan, who have always loved, encouraged, and supported me in every aspect of life. Finally, a very special thank you to my fiancé, Nicholas Hess, for providing a source of stress relief, making me laugh, and providing endless inspiration throughout the course of this project and my graduate career. 


\section{TABLE OF CONTENTS}

\section{Page}

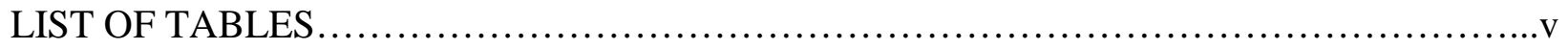

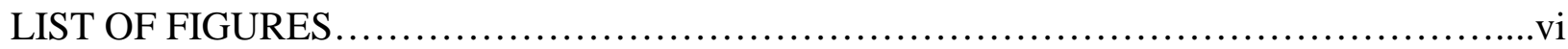

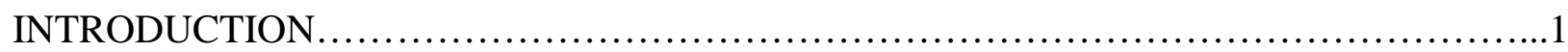

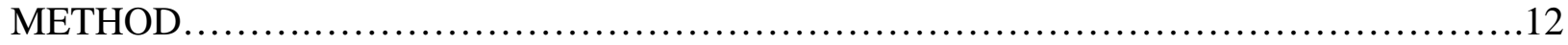

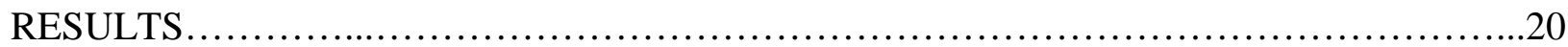

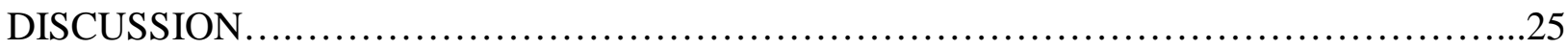

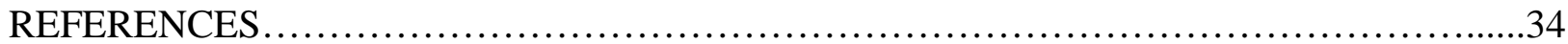

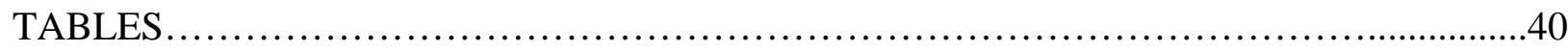

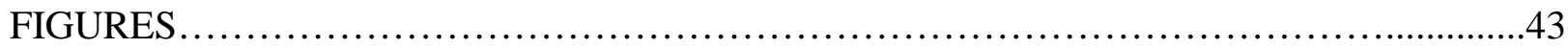




\section{LIST OF TABLES}

Page

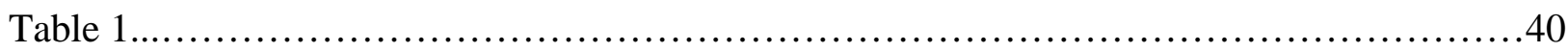

Statistical analyses for all outcomes measures.

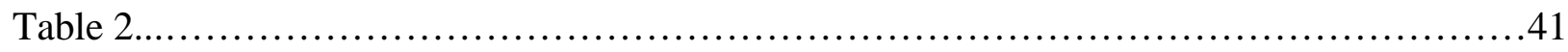

Percent larger-reinforcer choice, indifference points (IPs), and area under the curve (AUC) at baseline.

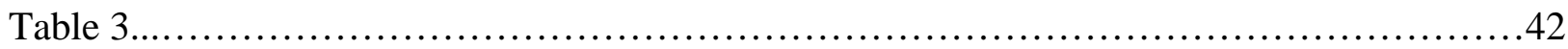

Indifference points (IPs) and area under the curve (AUC) across doses of acute nicotine. 


\section{LIST OF FIGURES}

Page

Figure 1

Mean sessions to reach baseline stability for singly and pair-housed rats.

Figure 2

Percent larger-reinforcer choice as a function of delay to the larger reinforcer for singly and pair-housed rats at baseline.

Figure 3

Mean area under the curve (AUC) for singly and pair-housed rats at baseline.

Figure 4

Mean indifference points (IPs) for singly and pair-housed rats at baseline.

Figure 5

Percent larger-reinforcer choice as a function of delay to the larger-reinforcer for singly and pair-housed rats following all doses of acute nicotine.

Figure 6

Mean area under the curve (AUC) for singly and pair-housed rats following all doses of acute nicotine.

Figure 7

Mean indifference points (IPs) for singly and pair-housed rats following all doses of acute nicotine. 
Effects of Housing Condition and Acute Nicotine on Impulsive Choice in

Lewis and Fischer 344 Rats

Impulsive behavior plays a role in many psychological and behavioral disorders, including substance abuse, obesity, and pathological gambling. There is also a section of the Diagnostic and Statistical Manual of Mental Disorders-V dedicated to impulse-control disorders (American Psychiatric Association [APA], 2013). Because impulse-control disorders can negatively affect quality of life, it is important to study the various mechanisms contributing to these maladaptive behaviors. Biological and environmental components underlying impulsive behavior should be investigated in order to develop better prevention and treatment methods for such disorders. It is also critical to examine how different psychoactive substances alter impulsive behavior given that substance-use disorders have an impulse-control component (APA, 2013).

\section{Impulsive Choice and Delay Discounting}

Impulsive choice is operationally defined as choosing a smaller, more immediate reinforcer over a larger, delayed reinforcer. A choice to smoke cigarettes now for immediate satisfaction is considered impulsive, as opposed to abstaining for a healthy future. Selfcontrolled choice is defined as choosing a larger, delayed reinforcer over a smaller, more immediate reinforcer. In the above example, self-controlled choice would be to abstain from smoking for future health. One way impulsive choice can be examined in the laboratory is through a behavioral procedure called delay discounting. This procedure involves discrete-trials choice between a smaller, more immediate reinforcer (e.g., one food pellet delivered immediately) and a larger, delayed reinforcer (e.g., three food pellets delivered after a delay). Delay-discounting procedures involve two independent variables: reinforcer delay and reinforcer 
magnitude. Either, or both, of these variables can be manipulated to assess changes in choice between the two alternatives. Biology, environment, and drugs can be studied as additional independent variables by manipulating species/strain, housing condition, or drug doses and examining effects on delay discounting.

There are various procedures for studying delay discounting in the lab, including adjusting reinforcer delay following each choice (e.g., Holt, Green, \& Myerson, 2012; Perry, Stairs, \& Bardo, 2008), adjusting reinforcer magnitude following each choice (e.g., Holt et al., 2012; Stein, Pinkston, Brewer, Francisco, \& Madden, 2012), changing delays across sessions (e.g., Madden, Smith, Brewer, Pinkston, \& Johnson, 2008; Wilhelm \& Mitchell, 2009), or changing delays across blocks of trials within a single session (e.g., Anderson \& Diller, 2010; Evenden \& Ryan, 1996; Kayir, Semenova, \& Markou, 2014). In adjusting-delay procedures, the delay to the larger reinforcer is adjusted based on the previous choice, while the delay to the smaller reinforcer and reinforcer magnitudes are held constant (Holt et al., 2012; Perry et al., 2008). If choice is for the smaller reinforcer in any given trial, the delay to the larger reinforcer is reduced until changes from impulsive choice to self-controlled choice are observed (Holt et al., 2012; Perry et al., 2008). Adjusting-amount procedures adjust the smaller, or larger, reinforcer magnitude based on the previous choice, while holding the delays and opposite reinforcer magnitude constant (e.g., Holt et al., 2012; Stein et al., 2012). For example, if choice is for the smaller reinforcer in any given trial, the larger reinforcer magnitude is increased until choice switches to the self-controlled alternative (e.g., Holt et al., 2012; Stein et al., 2012). The adjusting procedures allow either delay or amount to be titrated to a stable value, upon which effects of other variables can be assessed. 
Changing delays across sessions involves behavior coming in contact with one specific delay for an entire session. The delay to the larger reinforcer is increased in the next session, or after stability is reached (Madden et al., 2008; Wilhelm \& Mitchell, 2009). This allows a delaydiscounting function to be generated, which shows percent larger-reinforcer choice as a function of reinforcer delay. Adjusting the delay to the larger reinforcer across blocks of trials within a single session (within-session procedure) involves behavior coming in contact with a certain delay for a specified number of trials within a single block (e.g., Anderson \& Diller, 2010; Evenden \& Ryan, 1996). Evenden and Ryan (1996) developed this procedure involving forcedexposure and free-choice trials. Forced-exposure trials include one response alternative at a time, and are incorporated to ensure that behavior is exposed to both alternatives. Free-choice trials include two simultaneous response alternatives and choice is recorded over a number of trials. In the initial block of trials, the delay to both reinforcer magnitudes is generally $0 \mathrm{~s}$. After the block is completed (i.e., forced-exposure and free-choice trials), the delay to the larger reinforcer is increased and behavior (e.g., lever pressing) comes in contact with this new delay for the same number of trials as the previous block. Throughout the procedure, the delay to the smaller reinforcer is held constant (typically at $0 \mathrm{~s}$ ). A significant advantage of using the within-session procedure is that changes from impulsive choice to self-controlled choice, or vice versa, can be obtained for each session. Thus, a delay-discounting function can be generated for each session.

In the within-session procedure, percent larger-reinforcer choice is the primary dependent variable (e.g., Anderson \& Diller, 2010; Evenden \& Ryan, 1996; Kayir et al., 2014). It is calculated as the number of free-choice responses made on the lever associated with the larger, delayed reinforcer in a specific block, divided by the total number of free-choice responses made in that block. When both delays are $0 \mathrm{~s}$, choice is typically exclusive for the larger reinforcer. As 
the delay to the larger reinforcer is increased across blocks, percent larger-reinforcer choice declines, and choice switches to the smaller, more immediate reinforcer. This generates a delaydiscounting function, where percent larger-reinforcer choice is a function of the delay to the larger reinforcer. Beginning with a baseline delay-discounting function allows for experimental manipulation and examination of changes relative to baseline behavior.

The delay duration when choice switches from the larger, delayed reinforcer to the smaller, more immediate reinforcer is called an indifference point (IP) (Mazur, 1987). At this point, choice is for the smaller, more immediate reinforcer and larger, delayed reinforcer with equal frequency. When using the within-session procedure, IPs are often interpolated from percent larger-reinforcer data and fit to a function to determine the delay duration when choice for each reinforcer option is 50\% (e.g., Anderson \& Diller, 2010; Anderson \& Woolverton, 2005). Shorter IPs indicate more delay discounting (i.e., more impulsive choice) and longer IPs indicate less delay discounting (i.e., more self-controlled choice). Area under the delaydiscounting curve (AUC) can also be examined using the method proposed by Myerson, Green, and Warusawitharana (2001; see data analysis for further description). Smaller AUC indicates more delay discounting and larger AUC indicates less delay discounting.

\section{Physiological Contributors}

There is considerable evidence that dopamine (DA) and serotonin (5-HT) play a role in delay discounting with non-human animals (Mobini, Chiang, Al-Ruwaitea, Ho, Bradshaw, \& Szabadi, 2000; Winstanley, Dalley, Theobald, \& Robbins, 2003; Winstanley, Theobald, Dalley, Cardinal, \& Robbins, 2006). For example, to examine the role of DA and 5-HT in delay discounting, Winstanley et al. (2006) performed in vivo microdialysis in rats during a delaydiscounting task. 5-HT increased in the medial prefrontal cortex during task performance, while 
DA increased in the medial prefrontal cortex and orbitofrontal cortex simultaneously. These results suggest that 5-HT and DA systems in the prefrontal cortex are both involved in the regulation of choice. Studies by Winstanley et al. (2003) and Mobini et al. (2000) support the conclusion that regulation of DA and 5-HT is necessary for impulse control. Thus, examining delay discounting in rat strains that differ in DA and 5-HT levels may lend further information toward effects of DA and 5-HT on impulsive choice.

Two rat strains that may contribute to understanding biological influence on impulsive choice are Lewis (LEW) and Fischer 344 (F344) rats. LEW rats have fewer DA transporters and receptors in the nucleus accumbens and striatum relative to F344 rats (Flores, Wood, Barbeau, Quirion, \& Stivastava, 1998). LEW rats also have less 5-HT binding in the hippocampus, prefrontal cortex, and nucleus accumbens than F344 rats (Selim \& Bradberry, 1996) among other physiological differences (Selim \& Bradberry, 1996). Interestingly, indicated by shorter IPs and smaller AUC, choice is more impulsive for LEW rats than F344 rats across several delaydiscounting procedures when individually housed (Anderson \& Diller, 2010; Anderson \& Woolverton, 2005; Huskinson \& Anderson, 2012; Huskinson, Krebs, \& Anderson, 2012; Madden et al., 2008; Stein et al., 2012, also see Wilhelm \& Mitchell, 2009 and Richards et al., 2013 for exceptions), which gives support for a biological component in delay discounting. Thus, studying delay discounting in these rat strains may help elucidate a role of biology in impulsive choice (e.g., Anderson \& Diller, 2010; Anderson \& Woolverton, 2005).

\section{Environmental Contributors}

In addition to biological variables, impact of environmental condition on delay discounting can be considered. Environmental factors (e.g., housing condition) can be manipulated to assess effects on delay discounting (Kirkpatrick, Marshall, Clarke, \& Cain, 2013; 
Kirkpatrick, Marshall, Smith, Koci, \& Park, 2014; Perry et al., 2008). For example, Kirkpatrick et al. (2013) and Kirkpatrick et al. (2014) initially presented discrete-trials choice between one food pellet delivered after $10 \mathrm{~s}$ and one food pellet delivered after $30 \mathrm{~s}$ to Sprague-Dawley rats housed in enriched (i.e., group housed with toys and pulp paper bedding) and impoverished (i.e., singly housed in hanging wire cages with no toys and a wire mesh floor) environments. Reinforcer magnitude associated with the 30-s delay was increased from one pellet to three pellets in one-pellet increments. Rats in the impoverished environment chose the one-pellet alternative, associated with the 10-s delay, over the larger-reinforcer option more often than rats in the enriched environment. This suggests that environmental enrichment can reduce impulsive choice and increase self-controlled choice in rats relative to impoverished housing.

Environmental conditions may also influence how drugs affect choice (Perry et al., 2008; Zhu, Bardo, Green, Wedlund, \& Dwoskin, 2007). For example, Perry et al. (2008) examined delay discounting with enriched and impoverished environmental conditions using SpragueDawley rats. The enriched condition consisted of housing rats together in a large group with novel objects (i.e., toys), and the impoverished condition consisted of singly housed rats in hanging wire cages, with no objects (similar to Kirkpatrick et al., 2013; 2014). At baseline, delay discounting of rats in the enriched condition was less than rats in the impoverished condition, indicating higher self-controlled choice for rats in the enriched environment. Following administration of $d$-amphetamine, delay discounting for rats in the enriched environment increased while delay discounting for rats in the impoverished environment decreased. Huskinson et al. (2012) also examined effects of $d$-amphetamine on delay discounting, using singly housed LEW and F344 rats. Rats were not housed in hanging wire cages as in Perry et al. (2008) and Kirkpatrick et al. (2013; 2014); rather, they were singly 
housed in standard plastic cages with paper bedding. At baseline, choice was more impulsive for LEW rats (i.e., more delay discounting) than F344 rats. Delay discounting decreased in LEW rats following acute $d$-amphetamine administration, but had no effect on delay discounting in F344 rats. Together, these results suggest that drug effects may be dependent upon baseline rates of delay discounting as well as environmental condition.

\section{Nicotine Effects on Delay Discounting}

Nicotine is the main psychoactive component found in tobacco products and is a commonly used substance. Bickel, Odum, and Madden (1999) used an adjusting-delay procedure to measure IPs for delay discounting in a comparison of smokers and nonsmokers. The delaydiscounting questionnaires were based on hypothetical-monetary and hypothetical-cigarette rewards. Smokers discounted all rewards (i.e., money and cigarettes) more than nonsmokers. Smokers also discounted cigarette rewards more than monetary rewards as a within-subjects comparison. Fields, Collins, Leraas, and Reynolds (2009) also examined delay discounting between smokers and nonsmokers, using an adjusting-amount procedure. Smokers discounted monetary rewards more than nonsmokers. It is unknown, however, whether chronic nicotine use increases delay discounting or if greater delay discounting at baseline increases the likelihood of smoking, but a correlation between the two is clear (Bickel et al., 1999; Fields, Collins, Leraas, \& Reynolds, 2009; Heyman \& Gibb, 2006). Using animal models allows for greater experimental control and examination of drug effects on delay discounting in a more controlled setting. For example, nicotine can be administered to rats acutely or chronically to assess differential effects on delay discounting.

Acute effects of nicotine on delay discounting in rats have been mixed (e.g., Anderson \& Diller, 2010; Dallery \& Locey, 2005; Mendez, Gilbert, Bizon, \& Setlow, 2012). For example, at 
baseline, using a within-session procedure, delay discounting was greater for singly housed LEW rats than singly housed F344 rats (Anderson \& Diller, 2010). Acute nicotine (administered subcutaneously twice per week, prior to experimental session) decreased delay discounting in both rat strains (i.e., increased self-controlled choice). However, nicotine decreased delay discounting at a lower dose for LEW rats $(0.3 \mathrm{mg} / \mathrm{kg})$ than F344 rats $(1.0 \mathrm{mg} / \mathrm{kg})$, suggesting that drug effects depend on baseline delay discounting and/or biology. However, in contrast, Dallery and Locey (2005) and Locey and Dallery (2009) found that acute nicotine administration (administered subcutaneously) increased delay discounting (i.e., increased impulsive choice) in singly housed Long-Evans rats using an adjusting-delay procedure. Mendez et al. (2012) also examined acute doses of nicotine, administered subcutaneously prior to experimental sessions, in singly housed Long-Evans rats using a within-session delay-discounting procedure. No significant effect was discovered between acute nicotine and delay discounting in Long-Evans rats. Results suggest that drug effects depend on baseline delay discounting, as well as biology, and discrepancies in acute nicotine effects on delay discounting may be due to differences between rat strains or procedures, which warrant the need for further research.

\section{Nicotine, Physiological, and Environmental Interactions}

Once nicotine reaches the brain, it binds to nicotinic acetylcholine receptors (Benowitz, 2009) and stimulates the release of several neurotransmitters, including acetylcholine (ACh), DA, and 5-HT (Benowitz, 2009; Summers \& Glacobini, 1995; Zhu et al., 2007). The importance of DA and 5-HT in delay discounting is known (Winstanley et al., 2003; 2006; Mobini et al., 2000), but nicotine also increases ACh levels, which is much less identified in delay discounting. To examine the role of ACh in delay discounting, Mendez et al. (2012) tested several drugs that target ACh’s two receptor subtypes (nicotinic and muscarinic): nicotine (nicotinic agonist), 
mecamylamine (nicotinic antagonist), oxotremorine (muscarinic agonist), scopolamine (muscarinic antagonist), and atropine (muscarinic agonist) using singly housed Long-Evans rats. Nicotine, mecamylamine, and oxotremorine had no effect on delay discounting. However, scopolamine and atropine increased delay discounting. This suggests that muscarinic receptors are involved in delay discounting, but does not clarify a role of nicotinic receptors. However, lack of nicotinic drug effects may depend on rat strain, given that others have reported significant increases (Anderson \& Diller, 2010) and decreases (Dallery \& Locey, 2005; Locey \& Dallery, 2009) in delay discounting following acute nicotine administration using different strains. There is very little known about the function of ACh in delay discounting at this time, which emphasizes the need for further research. In addition, it is unknown whether LEW and F344 rats differ in levels of muscarinic and/or nicotinic ACh receptors. However, differences in DA and 5HT levels in LEW and F344 rats are known (Flores et al., 1998; Selim \& Bradberry, 1996), and these biological variables may interact with nicotine to alter delay discounting.

Cadoni, Muto, and Chiara (2009) and Sziraki et al. (2001) observed DA transmission in singly housed LEW and F344 rats at baseline and following acute nicotine administration. DA transmission was measured by implanting probes into the nucleus accumbens shell and core, and performing dual-probe microdialysis. At baseline, LEW and F344 rats did not differ in DA transmission. Acute nicotine increased DA transmission in both rat strains; however, DA transmission in the nucleus accumbens increased to a greater degree for LEW rats when compared to F344 rats. DA release occurred more rapidly and peaked at higher concentrations for LEW rats than F344 rats. Zeeb, Soko, Ji, and Fletcher (2016) suggest that the nucleus accumbens is involved in delay discounting. They performed in vivo microdialysis in rats and determined that DA release in the nucleus accumbens increased during a delay-discounting task. 
Thus, because the nucleus accumbens has been observed to be involved in delay discounting (Zeeb et al., 2016), it seems as though differences in DA turnover in the nucleus accumbens (Cadoni et al., 2009; Sziraki et al., 2001) may contribute to differential effects of nicotine on delay discounting in LEW and F344 rats. Given that nicotine increases extracellular DA levels, and higher DA levels are associated with less delay discounting (i.e., less impulsive choice), these results suggest that nicotine will reduce delay discounting to a larger degree in LEW rats than F344 rats.

Environmental enrichment may also modify effects of nicotine (Green, Cain, Thompson, \& Bardo, 2003; Zhu et al., 2007). For example, Zhu et al. (2007) examined effects of acute nicotine on DA clearance in the striatum and medial prefrontal cortex with Sprague-Dawley rats housed in enriched and impoverished environments. The enriched environment consisted of housing 8-12 rats in a large metal cage with several novel objects and the impoverished environment consisted of singly housing rats in hanging wire cages, with no objects (similar to Kirkpatrick et al., 2013; 2014 and Perry et al., 2008). Rats in the enriched condition were also handled each day, while rats in the impoverished condition were not handled. At baseline, there was no significant difference in DA clearance between rats in the two environmental conditions. However, following acute nicotine administration, rats in the enriched environment had an increase in DA clearance in the medial prefrontal cortex while rats in impoverished environments had no change in DA clearance relative to baseline. Given the role of the prefrontal cortex in delay discounting (Winstanley et al., 2003; 2006), it seems as though differences in DA clearance in the prefrontal cortex following nicotine administration may lead to differential effects of nicotine on delay discounting in impoverished and enriched housing conditions. Thus, because it is known that stimulants (e.g., nicotine) increase DA levels, it seems 
as though rats in enriched environments would have more DA clearance than rats in impoverished environments and in turn, nicotine would reduce delay discounting to a greater degree in rats in impoverished environments.

Another example of environmental influence on drug effects is from Green et al. (2003). Locomotor activity was measured in Sprague-Dawley rats, in enriched and impoverished environments. The enriched environment included housing 8-10 rats per large cage and plastic toys, which were replaced with novel objects each day. The impoverished environment included no toys, and rats were singly housed in hanging wire cages (similar to Kirkpatrick et al., 2013; 2014, Perry et al., 2008, and Zhu et al., 2007). Rats in the enriched environment were less active compared to rats in the impoverished environment at baseline and following acute nicotine administration. This suggests that environmental condition may have reduced stimulant effects of nicotine on locomotor activity. Greater locomotor activity has been correlated with greater delay discounting (i.e., more impulsive choice; Kirkpatrick et al., 2013; 2014). Therefore, environmental enrichment in LEW and F344 rats may also reduce effects of nicotine on delay discounting.

\section{Rationale}

Biological factors (e.g., Anderson \& Diller, 2010; Huskinson et al., 2012), environmental conditions (e.g., Kirkpatrick et al., 2013; 2014; Perry et al., 2008), and nicotine (e.g., Anderson \& Diller, 2010; Dallery \& Locey, 2005) influence delay discounting. Because environmental enrichment generally has two simultaneous components: social stimulation (i.e., group housing) and enrichment (i.e., toys), it is unknown whether one of these components has a greater effect on delay discounting. Therefore, in the current design, LEW and F344 rats were pair-housed, with no toys, to assess effects of social stimulation. In addition, biological and environmental 
factors may interact with nicotine to affect delay discounting (e.g., Cadoni et al., 2009; Zhu et al., 2007). Therefore, it was unclear whether effects of nicotine on delay discounting in singly housed (SH) LEW and F344 rats (Anderson \& Diller, 2010) would maintain when they were housed in littermate pairs as part of environmental enrichment. Because there is minimal research on acute effects of nicotine, and the literature that does exist is conflicting, there is a need for further research.

Considering evidence with SH LEW and F344 rats (e.g., Anderson \& Diller, 2010; Huskinson et al., 2012) and environmental effects (e.g., Perry et al., 2008; Zhu et al., 2007), four outcomes were hypothesized: (1) delay discounting would be greater in LEW rats than F344 rats at baseline regardless of housing condition, (2) delay discounting would be greater in SH rats (from Anderson \& Diller, 2010) than pair-housed (PH) rats, regardless of strain, at baseline (3) delay discounting would decrease for all rats following acute nicotine administration, (4) delay discounting would decrease to a larger degree for $\mathrm{SH}$ rats than $\mathrm{PH}$ rats following acute nicotine administration (i.e., SH rats would be more sensitive to effects of nicotine on delay discounting), and (5) the largest decrease in delay discounting following acute nicotine administration would be for SH LEW rats, followed by SH F344 rats, followed by PH LEW rats, and finally PH F344 rats.

\section{Method}

The procedure outlined systematically replicated the procedure described by Anderson and Diller (2010). Any procedural variations are noted where applicable. All procedures were approved by the Animal Care and Use Committee at West Virginia University. 


\section{Subjects}

Six experimentally naïve, male LEW rats and six experimentally naïve, male F344 rats served as PH subjects. All PH rats were housed in littermate pairs in standard plastic cages with paper bedding. Data from PH rats were compared to archival data from SH rats, taken from Anderson and Diller (2010). SH rats were eight male LEW and seven male F344 rats and were singly housed in identical, standard plastic cages with paper bedding. All rats were obtained from Harlan Sprague-Dawley, Inc. (Indianapolis, IN) and were six weeks old when shipped. Rats were fed ad libitum for three weeks following shipment, began food restriction at nine weeks, and were 11 weeks old at the beginning of the experiment. Throughout the experiment, rats had continuous access to water and were housed with controlled environmental conditions (temperature, $24^{\circ} \mathrm{C} ; 12$-h reverse light/dark cycle). Sessions were conducted at the same time each day, five days per week (Monday-Friday). Rats were fed 13-15 g of food, approximately 30 min following each session. For $\mathrm{PH}$ rats, this was done by placing approximately $26-30 \mathrm{~g}$ of food in each shared cage. Rats were food restricted for approximately $22 \mathrm{~h}$ afterward. As reported by Anderson and Diller (2010), SH LEW and F344 rats weighed approximately $300 \mathrm{~g}$ at the start of experimental testing. At the start of the current experiment, weights ranged from 263$317 \mathrm{~g}$ for PH LEW rats and 239-268 g for PH F344 rats. At the end of experimental testing, weights ranged from 423-520 g for PH LEW rats and 382-462 g for PH F344 rats.

\section{Apparatus}

Experimental sessions were conducted in eight standard operant-conditioning chambers for rats, each enclosed in a melamine sound-attenuating cubicle (Med Associates, VT). Each chamber contained a working area of $30.5 \mathrm{~cm}$ by $24.5 \mathrm{~cm}$ by $21.0 \mathrm{~cm}$, a grid floor, and a 45-mg pellet dispenser with a pellet receptacle centered between two retractable response levers. Levers 
were $11.5 \mathrm{~cm}$ apart from each other and required at least $0.25 \mathrm{~N}$ of force for a response to be recorded. Levels were $4.8 \mathrm{~cm}$ wide, protruded $1.9 \mathrm{~cm}$ into the chamber, and were elevated $8 \mathrm{~cm}$ from the grid floor. Two 28-V stimulus lights, $2.5 \mathrm{~cm}$ in diameter, were placed approximately 7 cm above each lever. Each chamber had a $28 \mathrm{~V}$ houselight on the wall opposite to the working wall, and a ventilation fan to circulate air and to mask extraneous noise. Data collection and programmed consequences were controlled by a personal computer equipped with Med-PC software (Med Associates, VT).

\section{Procedure}

Lever-press training. Prior to lever-press training, food pellets were delivered on a variable-time (VT) 60-s schedule for a single session. Both levers were retracted from the chamber and the session ended following 60 food-pellet deliveries. In the course of lever-press training, both levers were extended into the chamber and pellets were delivered according to a tandem fixed-ratio (FR) 1 differential-reinforcement-of-other-behavior (DRO) 10-s resetting schedule. Sessions were terminated after 8 h. If lever pressing was not acquired during this session, a conjoint VT 60 s FR 1 schedule was in effect for five sessions. After five sessions, if lever pressing had not been acquired, it was shaped by successive approximations.

After lever pressing was acquired, an alternating FR 1 schedule went into effect. During each session, one lever was extended into the chamber, with a cue light illuminating over it. A press on the extended lever resulted in one food pellet following one response. The light and FR 1 contingency alternated between the two levers after every five food pellets were delivered, and the session ended following 40 food-pellet deliveries. Sessions continued until lever pressing was consistent on both levers. 
Delay-discounting procedure. All sessions began with a 10-min blackout period, followed by five blocks of eight trials each. After the blackout, the houselight was illuminated and remained lit for the remainder of the session. Each block began with two forced-exposure trials, followed by six free-choice trials. Each block was signaled by the presence of the houselight. During each forced-exposure trial, one random lever was extended into the chamber and the cue light above it was illuminated. After one response, the lever retracted, and one or three food pellets were delivered either immediately or after a delay, dependent upon the magnitude of reinforcement and reinforcer delay associated with the lever. During the second forced-exposure trial, the alternative lever was extended into the chamber and the same process occurred with the other reinforcer magnitude and delay. The houselight flashed for $0.1 \mathrm{~s}$ as each food pellet was delivered. Groups were counterbalanced, such that half of each group received the three-pellet option for presses on the right lever, and half received the three-pellet option for presses on the left lever. Levers correlated with each pellet option (one or three food pellets) remained constant throughout the experiment.

During free-choice trials, both levers were extended with corresponding cue lights, and choice for each lever was recorded. After a response on one lever was made, the cue lights turned off, both levers retracted, and food was delivered either immediately (one food pellet) or after a delay (three food pellets), dependent upon which lever was pressed. The houselight flashed for $0.1 \mathrm{~s}$ either one or three times, as each food pellet was delivered. Each new trial began every $100 \mathrm{~s}$, resulting in varying inter-trial intervals (ITI).

If a response did not occur within $30 \mathrm{~s}$ of trial onset for either trial type (i.e., forced or free), it was considered an omission. At this point, the cue light(s) turned off, lever(s) retracted, and an ITI began. The next trial began $100 \mathrm{~s}$ from the beginning of the omission trial (ITI $=70$ 
s). If six or more omissions occurred during a given session, the data from that session were not included in data analyses.

Establishing baseline. Prior to drug manipulation, a baseline delay-discounting function was established for each rat. Delay to food presentation increased across blocks of trials. Initially, during each block, an FR 1 contingency was in effect for presses on both levers, with no delay associated with presses on either lever. After at least $80 \%$ of responses during free-choice trials were for the larger reinforcer for one session, the delay to the larger reinforcer was increased across blocks according to the sequence: 0, 1, 2, 4, and 8 s. Again, after at least $80 \%$ of responses for the larger reinforcer were observed during free-choice trials, the delay to the larger reinforcer was increased to the sequence: $0,2,4,8$, and $16 \mathrm{~s}$. This process was repeated until a delay-discounting function was obtained for each rat without floor (i.e., exclusive choice for the smaller, immediate reinforcer) and ceiling (i.e., exclusive choice for the larger, delayed reinforcer) effects. For example, the sequence was increased to 0, 5, 10, 20, and $40 \mathrm{~s}$ as needed.

A minimum of ten sessions at the terminal delay series were conducted to obtain a baseline delay-discounting function. Each session consisted of 40 total trials (10 forcedexposure and 30 free-choice). Sessions continued until stability criteria were reached, which included: no increasing or decreasing trends in total percent larger-reinforcer choice across the last five sessions, mean percent larger-reinforcer choice at least $80 \%$ for the 0 -s block across the last five sessions, and no more than $20 \%$ variation in total percent larger-reinforcer choice during the last five sessions. In Anderson and Diller (2010), 0-s probe sessions were conducted once per week (on Wednesdays) to test for sensitivity to variations in reinforcer delay and reinforcer magnitude. During these sessions, all delay values were set to $0 \mathrm{~s}$ for all blocks within the session and percent larger-reinforcer choice was recorded. Criteria for 0-s probe sessions 
included at least $80 \%$ total larger-reinforcer choice and at least 4/6 larger-reinforcer choices per block. If criteria were not met, additional probe sessions were conducted until criteria were met. Once criteria were met, the baseline increasing-delay sequence was reinstated. However, in the current study, responding showed significant carryover effects following 0-s probe sessions, preventing establishment of a stable baseline; thus, they were eliminated from the current procedure following ten weeks (i.e., 50 total sessions). Probe sessions were reinstated as needed for individual rats when percent larger-reinforcer choice approached floor.

Acute nicotine administration. After establishing stable baseline delay-discounting functions, acute effects of nicotine on percent larger-reinforcer choice were examined. Nicotine and its vehicle control (saline) were administered subcutaneously to all rats immediately prior to each session. Before administration of nicotine, injections of saline were given to all rats for at least two sessions to test for behavioral interference due to injection procedures. Control days were Mondays and Thursdays before each test. Nicotine or vehicle administrations occurred on Tuesdays and Fridays of each week, given that percent larger-reinforcer choice was at least $80 \%$ for the most recent free-choice 0-s block (control day) and total percent larger-reinforcer choice was within the range of the last five baseline sessions. All doses of nicotine $(0.1,0.3,1.0 \mathrm{mg} / \mathrm{kg})$ were administered in descending order, with each dose administered a minimum of twice. Additional doses were administered if substantial variability in choice occurred between the two administrations. This allowed for development of individual-subject acute dose-response functions. Data from drug-administration sessions were omitted from data analyses if percent larger-reinforcer choice was not at least $80 \%$ during free-choice trials in the 0 -s block.

There were a few instances where baseline shifted following administration of the first nicotine dose $(1.0 \mathrm{mg} / \mathrm{kg})$. When this happened, drug testing was suspended and a 0-s probe 
session was conducted. Subsequent 0 -s probe sessions were conducted if criteria for probe sessions were not met during the initial session. Following 0-s probes, baseline was reestablished for a minimum of five sessions before drug testing resumed. Stable delay discounting for two rats (one LEW and one F344) was re-established for a minimum of ten sessions on a different delay series than established during the baseline phase. When this occurred, data from drug-administration sessions prior to re-establishing baseline were not included in data analyses.

Drugs. Each dose of nicotine was delivered in a $0.9 \%$ saline vehicle at a concentration of $1.0 \mathrm{mg} / \mathrm{mL}$. Doses were delivered in a volume of $1.0 \mathrm{~mL} / \mathrm{kg}$. Nicotine hydrogen tartrate was purchased from Sigma-Aldrich (St. Louis, MO).

\section{Data Analysis}

The primary dependent variable was percent larger-reinforcer choice. Percent largerreinforcer choice was calculated by dividing the number of free-choice responses on the lever associated with three food pellets available after a delay, by the total number of free-choice responses made in each block. Calculating percent larger-reinforcer choice across delays allowed for establishment of a baseline delay-discounting function. Percent larger-reinforcer choice was plotted as a function of the delay to the larger reinforcer and was then used to interpolate IPs for each subject and calculate AUC. IPs were interpolated from the percent larger-reinforcer choice data by using a nonlinear regression to fit a logistic equation used in previous experiments (e.g., Anderson \& Diller, 2010; Huskinson et al., 2012). Longer IPs indicate more delay discounting, and shorter IPs indicate less delay discounting.

AUC was calculated according to the formula described by Myerson et al. (2001). AUCs could range from 0.0 (exclusive choice for the smaller, immediate reinforcer) to 1.0 (exclusive choice for the larger, delayed reinforcer). Smaller AUCs indicate more delay discounting, and 
larger AUCs indicate less delay discounting. This is consistent with data analyses of previous studies (e.g., Anderson \& Diller, 2010; Huskinson et al., 2012). Given that rats' choice stabilized on different delay series, AUC values were also used to evaluate functional equivalence across delay series. Number of sessions required to reach stability were also calculated and compared across housing type and rat strain.

Data collected from PH rats were compared to archival data of SH rats, collected by Anderson and Diller (2010), to examine housing effects. Data were compared at baseline, and following all doses of acute nicotine. At baseline, a 2x2x2 factorial analysis of variance (ANOVA) was used to examine main effects and interactions of housing condition, rat strain, and delay series on IPs and AUC. Tukey's Honestly Significant Difference (HSD) test was used to make pair-wise comparisons as needed. Following acute nicotine administration, a mixed ANOVA was used to examine IPs and AUC. Between-subject variables were housing condition (2 levels), rat strain (2 levels), and delay series (2 levels) and within-subject variables were various doses of nicotine (4 levels). Huynh-Feldt corrections were used to adjust for violations of the sphericity assumption, and Tukey's HSD test was used to make pair-wise comparisons between means. For one PH LEW rat (JOL4), percent larger-reinforcer choice dropped below $80 \%$ during the 0 -s block at the highest nicotine dose $(1.0 \mathrm{mg} / \mathrm{kg})$. Therefore, for this rat, a mean imputation was conducted using the mean of the other five PH LEW rats at $1.0 \mathrm{mg} / \mathrm{kg}$ nicotine. Statistical analyses were conducted with and without data from this individual rat and did not affect results. For all analyses, statistical significance was defined as $p<.05$.

\section{Results}

Statistical analyses for all outcome measures are shown in Table 1. 


\section{Baseline}

All sessions that occurred following lever-press training and prior to the first saline injection were included in the baseline phase. Figure 1 shows that PH LEW and F344 rats required significantly fewer days to meet baseline stability criteria than SH LEW and F344 rats. PH LEW rats required a mean of $83.0($ SEM $=16.35)$ sessions to reach stability while SH LEW rats required a mean of $150.4(S E M=2.06)$ sessions. In addition, PH F344 rats required a mean of $106.0(S E M=10.9)$ sessions to reach stability while SH F344 rats required a mean of 160.3 $(S E M=0.57)$ sessions. As reported by Anderson and Diller (2010), when singly housed, number of sessions to reach stability was significantly different for LEW and F344 rats, with F344 rats requiring fewer sessions. However, when pair-housed, this strain difference was not observed.

Percent larger-reinforcer choice. Figure 2 shows percent larger-reinforcer choice as a function of block in session (i.e., corresponding to the increasing delay across blocks) for the last five baseline sessions (filled symbols). As the delay to the larger reinforcer (i.e., three food pellets) increased across blocks, percent choice for the larger reinforcer declined, supporting delay discounting. All SH rats responded on a 40-s terminal delay series. However, PH rats responded on variable terminal delay series. Four out of six LEW rats responded on a 40-s terminal delay series, while two out of six responded on a 16-s terminal delay series. In addition, four out of six F344 rats responded on a 16-s terminal delay series, while two out of six responded on a 40-s terminal delay series. Terminal delay series for individual subjects are shown in Table 2.

Figure 2 also shows data from 0-s probe sessions, where the delay to the larger reinforcer was held constant at $0 \mathrm{~s}$ across all blocks within a session (unfilled circles). A direct comparison cannot be made between $\mathrm{SH}$ and $\mathrm{PH}$ rats during 0 -s probe sessions, given that 0 -s probes were 
discontinued after ten weeks for PH rats. However, percent larger-reinforcer choice shifted in a similar manner when 0-s probes were introduced in both studies, which is demonstrated by the deviating delay-discounting functions in Figure 2 (filled versus unfilled symbols). These results suggest that behavior was sensitive to reinforcer amounts and delays prior to nicotine administration. Overall, delay-discounting functions appear similar across housing type and rat strain, suggesting functional equivalence. However, two exceptions appear in Figure 2 and Table 2 for PH LEW and F344 rats, suggesting that functional equivalence of delay series was not achieved. Percent larger-reinforcer choice was greater for LEW rats responding on the 40-s delay series relative to the 16-s delay series. Conversely, percent larger-reinforcer choice was greater for F344 rats responding on the 16-s delay series relative to the 40-s delay series.

Area under the curve (AUC). Functional equivalence was also evaluated through statistical analysis of AUC. AUC values for individual subjects averaged over the last five baseline sessions are shown in Table 2. In addition, mean AUC values for the last five baseline sessions are displayed in Table 2 and Figure 3. To examine whether delay series significantly influenced AUC in PH rats, independent-samples $t$-tests were conducted to make pair-wise comparisons within strains. Presented in Figure 3, AUC was significantly larger for PH LEW rats responding on the 40-s delay series relative to PH LEW rats responding on the 16-s delay series, $t(4)=4.05, p=.016$. However, there was no significant difference between responding on the 40-s and 16-s delay series for PH F344 rats.

Also suggested by Figure 3, functional equivalence was demonstrated within housing conditions, which was supported by a significant interaction between housing condition and rat strain. Collapsed across delay series, mean AUC for PH LEW rats was $0.35(S E M=0.02)$ and for PH F344 rats, mean AUC was $0.37(S E M=0.02)$. These values were not significantly 
different from one another, supporting functional equivalence. For SH LEW rats, mean AUC was $0.10(S E M=0.01)$ and for SH F344 rats, mean AUC was $0.14(S E M=0.01)$. These values were not statistically significantly different from one another, also supporting functional equivalence. AUC was significantly larger for PH LEW rats than SH LEW rats, regardless of delay series. This difference was also observed for PH and SH F344 rats. Thus, paired housing significantly increased AUC relative to single housing.

Indifference points (IPs). Given that rats responded on different terminal delay series, IPs were used to make direct comparisons in delay discounting between rat strains and housing conditions. Different delay series contribute to values of IPs. That is, in functionally equivalent delay-discounting functions, a 16-s delay series will give a shorter IP than a 40-s delay series. IPs for individual subjects are shown in Table 2, while mean IPs across housing conditions and rat strains are displayed in Table 2 and Figure 4. Shown in Figure 4, and supported by a significant interaction between rat strain and housing condition, IPs for PH LEW rats $(M=8.80 \mathrm{~s}, S E M=$ $0.54)$ were significantly longer than SH LEW rats $(M=3.03 \mathrm{~s}, S E M=0.44)$. In addition, IPs for PH F344 rats $(M=7.67 \mathrm{~s}, S E M=0.54)$ were significantly longer than SH F344 rats $(M=4.48 \mathrm{~s}$, $S E M=0.48)$. Reported by Anderson and Diller (2010), there was also a significant strain difference between SH LEW and F344 rats, with SH F344 having significantly longer IPs than SH LEW rats. However, paired housing attenuated this strain difference.

\section{Acute Nicotine Administration}

Percent larger-reinforcer choice. Figure 5 shows percent larger-reinforcer choice as a function of the delay to the larger reinforcer for saline and each dose of acute nicotine. During acute nicotine administration, all SH rats responded on a 40-s terminal delay series. However, PH rats responded on variable delay series. Delay-discounting functions for two PH rats (JOL1 
and JOF2) approached floor during nicotine administration. For these two rats, delay series were reduced and baseline was reestablished prior to nicotine administration. Therefore, during nicotine administration, three out of six PH LEW rats responded on a 40-s terminal delay series and three out of six responded on a 16-s terminal delay series. In addition, five out of six PH F344 rats responded on a 16-s terminal delay series, while one out of six responded on a 40-s terminal delay series (shown in Table 3 and Figure 5).

Shown in Figure 5, acute nicotine dose dependently increased percent larger-reinforcer choice across rat strains, delay series, and housing conditions. Although percent larger-reinforcer choice increased, delay discounting still occurred. Increases in percent larger-reinforcer choice were more apparent at the two highest doses of nicotine (i.e., 0.3 and $1.0 \mathrm{mg} / \mathrm{kg}$ ) for PH rats relative to SH rats. For SH LEW rats, the largest increase was observed at the $0.3 \mathrm{mg} / \mathrm{kg}$ dose, and for SH F344 rats, the largest increase was observed at the $1.0 \mathrm{mg} / \mathrm{kg}$ dose. Conversely, for $\mathrm{PH}$ rats, increases in percent larger-reinforcer choice were apparent at 0.3 and $1.0 \mathrm{mg} / \mathrm{kg}$ doses regardless of strain.

Area under the curve (AUC). Individual-subject AUC values across doses of acute nicotine are shown in Table 3. In addition, mean AUC values across doses of acute nicotine are presented in Table 3 and Figure 6. Under control (non-drug) conditions, mean AUC for PH LEW rats responding on the 16-s delay series $(M=0.30, S E M=0.02)$ and for PH LEW rats responding on the 40-s delay series $(M=0.41, S E M=0.02)$ were not significantly different from PH F344 rats responding on the 16 -s delay series $(M=0.34, S E M=0.02)$ or 40 -s delay series $(M$ $=0.32, S E M=0.04)$. Shown in Figure 6, acute nicotine increased mean AUC to a larger degree in $\mathrm{PH}$ rats relative to $\mathrm{SH}$ rats, regardless of delay series. Collapsed across rat strain, mean AUC for all PH rats responding on the 16-s delay series across all doses of nicotine (saline: $M=0.29$, 
$S E M=0.02 ; 0.1 \mathrm{mg} / \mathrm{kg}: M=0.31, S E M=0.02 ; 0.3 \mathrm{mg} / \mathrm{kg}: M=0.43, S E M=0.02 ; 1.0 \mathrm{mg} / \mathrm{kg}: M$ $=0.66, S E M=0.04)$ and for PH rats responding on the 40 -s delay series across all doses of acute nicotine (saline: $M=0.30, S E M=0.03 ; 0.1 \mathrm{mg} / \mathrm{kg}: M=0.39, S E M=0.03 ; 0.3 \mathrm{mg} / \mathrm{kg}: M=0.49$, $S E M=0.03,1.0 \mathrm{mg} / \mathrm{kg}: M=0.50, S E M=0.06)$ were significantly larger than for $\mathrm{SH}$ rats following saline $(M=0.14, S E M=0.01), 0.1 \mathrm{mg} / \mathrm{kg}(M=0.12, S E M=0.01)$, and $0.3 \mathrm{mg} / \mathrm{kg}(M$ $=0.14, S E M=0.02$ ) nicotine administration.

Additionally, collapsed across rat strain and delay series, post-hoc tests revealed that mean AUC for PH rats was significantly larger following $1.0 \mathrm{mg} / \mathrm{kg}(M=0.58, S E M=0.04)$ and $0.3 \mathrm{mg} / \mathrm{kg}(M=0.46, S E M=0.02)$ than following saline $(M=0.29, S E M=0.02)$. Mean AUC was also significantly larger for PH rats as compared to SH rats within each nicotine dose. Average difference scores between housing conditions were $0.15(S E M=0.02)$ for saline, 0.38 $(S E M=0.02)$ for $0.1 \mathrm{mg} / \mathrm{kg}, 0.31(S E M=0.02)$ for $0.3 \mathrm{mg} / \mathrm{kg}$, and $0.24(S E M=0.03)$ for 1.0 $\mathrm{mg} / \mathrm{kg}$.

Indifference points (IPs). Individual-subject IPs (s) across doses of acute nicotine are shown in Table 3. In addition, mean IPs across doses of acute nicotine are displayed in Table 3 and Figure 7. Shown in Figure 7, and supported by a significant 3-way interaction between nicotine dose, housing condition, and rat strain, mean IPs following $1.0 \mathrm{mg} / \mathrm{kg}(M=14.58 \mathrm{~s}$, $S E M=1.95)$ and $0.3 \mathrm{mg} / \mathrm{kg}(M=11.85 \mathrm{~s}, S E M=1.27)$ for PH LEW rats were significantly longer than following saline administration $(M=7.19 \mathrm{~s}, S E M=0.83)$ for PH LEW rats. In addition, mean IPs following $1.0 \mathrm{mg} / \mathrm{kg}(M=14.96 \mathrm{~s}, S E M=2.62)$ and $0.3 \mathrm{mg} / \mathrm{kg}(M=13.78 \mathrm{~s}$, $S E M=1.71)$ for PH F344 rats were significantly longer than following saline $(M=5.65 \mathrm{~s}, S E M$ = 1.11) for PH F344 rats. Thus, when pair-housed, IPs were significantly increased at the two highest nicotine doses (1.0 and $0.3 \mathrm{mg} / \mathrm{kg}$ ) regardless of rat strain. These results are in contrast to 
SH rats, where $1.0 \mathrm{mg} / \mathrm{kg}$ significantly increased IPs for F344 rats and $0.3 \mathrm{mg} / \mathrm{kg}$ significantly increased IPs for LEW rats. This is also supported by a significant 2-way interaction between housing condition and nicotine dose. Collapsed across rat strain, mean IPs were significantly longer following $1.0 \mathrm{mg} / \mathrm{kg}(M=14.50 \mathrm{~s}, S E M=1.56)$ and $0.3 \mathrm{mg} / \mathrm{kg}$ for $\mathrm{PH}$ rats $(M=12.81 \mathrm{~s}$, $S E M=1.07)$ than following saline administration for $\mathrm{PH}$ rats $(M=6.42 \mathrm{~s}, S E M=0.70)$.

Together, these results suggest that nicotine had a more consistent effect across rat strains when pair housed relative to $\mathrm{SH}$ rats. In addition, mean IPs following $1.0 \mathrm{mg} / \mathrm{kg}$ and $0.3 \mathrm{mg} / \mathrm{kg}$ for PH rats were significantly longer than following $1.0 \mathrm{mg} / \mathrm{kg}(M=6.32 \mathrm{~s}, S E M=1.24)$ and $0.3 \mathrm{mg} / \mathrm{kg}$ $(M=5.06 \mathrm{~s}, S E M=0.81)$ for SH rats.

\section{Discussion}

The present experiment was designed to examine whether the previously reported strain difference in delay discounting between SH LEW and F344 rats (e.g., Anderson \& Diller, 2010; Huskinson et al., 2012) would maintain when rats were pair-housed as part of environmental enrichment. When singly housed, choice is more impulsive for LEW rats relative to F344 rats (e.g., Anderson \& Diller, 2010; Huskinson et al., 2012). Pair housing significantly reduced delay discounting (i.e., reduced impulsive choice) relative to SH rats, regardless of rat strain, at baseline. In addition, no strain difference was observed in delay discounting when rats were pairhoused.

Next, effects of acute nicotine on delay discounting were examined in PH LEW and F344 rats. Previously reported by Anderson and Diller (2010), acute nicotine significantly reduced delay discounting (indicated by increased IPs and AUC) at different doses for SH LEW and F344 rats (LEW: $0.3 \mathrm{mg} / \mathrm{kg}$; F344: $1.0 \mathrm{mg} / \mathrm{kg}$ ). However, during the current experiment, 0.3 and $1.0 \mathrm{mg} / \mathrm{kg}$ both significantly reduced delay discounting regardless of rat strain in PH rats. Given 
the present results, it seems as though previously observed strain differences in LEW and F344 rats may be due to differential effects of individual housing.

Only a few studies to date have examined influences of environmental enrichment on delay discounting (e.g., Kirkpatrick et al., 2013; 2014; Perry et al., 2008). However, the majority of these studies have focused on environmental enrichment in terms of two factors—social housing and novel objects (i.e., toys) in the environment. For example, Perry et al. (2008) studied delay discounting using male Sprague-Dawley rats and divided them into two groups—enriched (group housed with toys) and impoverished (singly housed with no toys) environments. They reported that choice for rats in the enriched condition was significantly more self-controlled than for rats in the impoverished environmental condition. Other studies have supported this finding using delay-discounting procedural variations (Kirkpatrick et al., 2013; 2014). Thus, the present study aimed to parse apart effects of social housing and novelty by examining delay discounting in PH rats, with no toys. Results suggest that the previously reported differences between rats in impoverished and enriched environments (e.g., Perry et al., 2008) are at least, in part, due to social versus isolated housing. Future research might be designed to evaluate effects of novelty alone in SH rats to determine whether it is a necessary and/or sufficient component of environmental enrichment.

\section{Physiological and Environmental Interactions}

LEW rats have fewer DA and 5-HT transporters and receptors in various brain regions, including the prefrontal cortex and nucleus accumbens, relative to F344 rats (Flores et al., 1998; Selim \& Bradberry, 1996). Delay discounting is also greater for LEW rats (i.e., more impulsive choice) relative to F344 rats when singly housed. To examine the role of DA and 5-HT in delay discounting, Winstanley et al. (2006) performed in vivo microdialysis in rats during a delay- 
discounting task. DA and 5-HT both increased in the medial prefrontal cortex during the task, indicating that both are involved in the regulation of choice. Other studies have supported this finding (Mobini et al., 2000; Winstanley et al., 2003). Due to the results obtained during the present study (i.e., there was no effect of rat strain on delay discounting when pair housed), it is possible that the previously reported strain difference in delay discounting between SH LEW and F344 rats (e.g., Anderson \& Diller, 2010; Huskinson et al., 2012) may be due to neurochemical changes induced by being housed in isolation.

In fact, there is evidence that individual housing has profound effects on various neurochemical responses in rats. For example, Garrido et al. (2013) examined concentrations of corticosterone and DA in the prefrontal cortex of male Wistar rats housed in enriched and impoverished environmental conditions. The enriched environment consisted of 10-12 rats in a large cage with toys, while the impoverished environment consisted of singly housed rats in standard plastic cages and no toys (in contrast to hanging wire cages as in Kirkpatrick et al., 2013; 2014, Perry et al., 2008; and Zhu et al., 2007). They found that rats in the impoverished condition had significantly larger corticosterone and dopaminergic responses (i.e., larger increases in both concentrations) to acute restraint stress relative to their enriched counterparts. In addition, Miura, Qiao, and Ohta (2002) found that F344 rats housed in isolation (i.e., singly housed in wire hanging cages and no toys) had significantly higher DA concentrations in the nucleus accumbens and 5-HT concentrations in the prefrontal cortex relative to F344 rats housed in a social environment (i.e., 2-3 rats per wire hanging cage with no toys). Given the roles of the nucleus accumbens (Zeeb et al., 2016) and prefrontal cortex (Winstanley et al., 2003; 2006) in delay discounting, it seems likely that being housed in isolation influences impulsive choice, at 
least in part, via these neurochemical mechanisms. Therefore, it is possible that the present results are partly due to a neurochemical change in LEW and F344 rats when pair-housed.

Conversely, several studies indicate that there are no differences in basal monoamine concentrations of rats between enriched and impoverished environmental conditions (Cadoni et al., 2009; Kirkpatrick et al., 2014; Jones, Hernandez, Kendall, Marsden, \& Robbins, 1992). Instead, it seems as though environmental enrichment alters functional turnover of monoamines (Jones et al., 1992; Miura et al., 2002; Zhu, Bardo, \& Dwoskin, 2013). For example, Kirkpatrick et al. (2014) used high-performance liquid chromatography (HPLC) to examine monoamine concentrations in the prefrontal cortex and nucleus accumbens of male Sprague-Dawley rats in impoverished and enriched environments. The impoverished condition consisted of singly housed rats in wire hanging cages with no toys, while the enriched condition consisted of 8-12 rats per large cage with novel objects. They found that basal monoamine levels did not differ between the two groups, although choice for rats in the enriched condition was more selfcontrolled relative to rats in the impoverished condition. Thus, these results are consistent with the finding that enrichment alters functionality of monoamines. In addition, Jones et al. (1992) used HPLC to examine whether social housing influenced DA transmission in various brain regions of male Lister hooded rats. They divided rats into isolated (i.e., singly housed with no toys) and social (i.e., six rats per cage with no toys) environmental conditions. They found that rats in a social environment had an increase in basal DA metabolism in the medial prefrontal cortex relative to rats housed in isolation but the groups did not differ in basal concentrations. Thus, it is possible that social housing alone contributes to effects of environmental enrichment on monoamine turnover (Stairs \& Bardo, 2009). 
It is also possible that differences in monoamine turnover between socially housed and isolated rats contributed to the differential effects of nicotine observed in the present experiment. In SH LEW and F344 rats, DA transmission in the nucleus accumbens increased to a greater degree for LEW rats compared to F344 rats (Cadoni et al., 2009; Sziraki et al., 2001). It is possible that differences in DA transmission between LEW and F344 rats are attenuated with social housing. In studies by Zhu et al. (2013) and Zhu et al. (2007), nicotine was administered to male Sprague-Dawley rats in enriched and impoverished environmental conditions. The enriched condition consisted of group housing in a large cage with toys while the impoverished condition consisted of single housing in wire hanging cages with no toys. They measured DA clearance by in vivo electrochemistry in the nucleus accumbens shell and core, as well as the prefrontal cortex, and found that nicotine had differential effects on DA clearance across housing conditions. That is, in the nucleus accumbens shell, nicotine increased DA clearance in rats in the enriched condition, but not in rats in the impoverished condition. Conversely, nicotine increased DA clearance in the nucleus accumbens core in rats in the impoverished condition, but not in rats in the enriched condition. In addition, rats in the enriched condition had a significant increase in DA clearance in the prefrontal cortex while rats in isolated environments had no change in DA clearance relative to baseline. It may be that this differential response to nicotine across housing conditions contributed to the observed differences in delay discounting during the present study. However, it is unclear why delay discounting in PH LEW and F344 rats was more sensitive to effects of nicotine relative to SH rats, given that social housing is often described as an enrichment procedure (Stairs \& Bardo, 2009).

Rather, it may be the case that effects of nicotine on delay discounting are baseline dependent. In SH LEW and F344 rats, choice was more impulsive for LEW rats at baseline and 
nicotine reduced delay discounting at a lower dose relative to F344 rats. In the present study, there were no baseline differences in delay discounting for PH LEW and F344 rats, and in turn, there were no differences in nicotine’s effects on delay discounting. Perry et al. (2008) found that $d$-amphetamine administration had differential effects on Sprague-Dawley rats in enriched and impoverished environmental conditions. The enriched environment consisted of group housing in a large cage with toys while the impoverished environment consisted of single housing in hanging wire cages and no toys. $d$-Amphetamine dose dependently reduced delay discounting (i.e., reduced impulsive choice) in rats in the impoverished condition and increased delay discounting (i.e., increased impulsive choice) in rats in the enriched condition. This suggests that DA may play a central role in baseline-dependent drug effects. Nicotine was administered in the present study, and although it acts primarily at nicotinic ACh receptors and stimulates release of ACh, it has downstream effects on the release of DA and 5-HT (Benowitz, 2009). Thus, it is possible that the current results are evidence of baseline dependency. Future research could be designed to evaluate administration of a DA antagonist in concert with nicotine to help determine whether action at DA receptors is essential for baseline dependency.

\section{Limitations}

Although the same stability criteria were used in the present experiment and its comparison study (i.e., Anderson \& Diller, 2010), it is possible that a few minor procedural differences contributed to the obtained results. First, 0 -s probe sessions were conducted once per week during Anderson and Diller (2010) throughout baseline. In the present study, choice showed significant carryover effects following 0 -s probe sessions and were thus discontinued following ten weeks (i.e., 50 sessions). Perry et al. (2008) found that days to stability on a delaydiscounting task did not differ between enriched (i.e., group housing with toys) and 
impoverished (i.e., single housing in hanging wire cages with no toys) environmental conditions. Thus, reducing the number of 0 -s probe sessions may be an underlying reason as to why $\mathrm{PH}$ rats required significantly fewer days to reach baseline stability relative to SH rats in the current study. However, Perry et al. (2008) used an adjusting-delay procedure and included toys in the enriched environment, which may contribute to why differences were seen in the present study and not during Perry et al. (2008).

In addition, different durations of exposure to experimental contingencies may have contributed to the differences observed at baseline between SH and PH rats. According to Aparicio, Elcoro, and Alonso-Alvarex (2015), who conducted a long-term study of delay discounting in LEW and F344 rats, differences in delay discounting between these two rat strains changed as a function of exposure to the contingencies, and choice converged. They concluded that impulsive choice is dynamic and changes throughout the course of learning. Thus, different amounts of exposure across housing conditions during the present study may have contributed to the observed difference between SH and PH rats. The present experiment evaluated baseline stability via pre-determined stability criteria. Future research may be designed to equate exposure to contingencies during baseline to help determine whether differences between housing conditions remain.

In addition, although identical baseline stability criteria were used in both studies, steeper baseline delay discounting was obtained during Anderson and Diller (2010) than during the present experiment. Baseline delay discounting during Anderson and Diller (2010) approached floor, regardless of rat strain. If the terminal delay sequence was reduced to $16 \mathrm{~s}$ for $\mathrm{SH}$ rats, it is likely that obtained delay-discounting functions would have been more similar to those obtained in the present experiment. However, using IPs as a primary dependent measure helps reduce 
effects of delay series on measures of delay discounting. Because IPs were significantly longer for $\mathrm{PH}$ rats relative to $\mathrm{SH}$ rats, it seems unlikely that floor effects contributed to the observed effect of housing.

In the present study, nicotine reduced delay discounting to a greater degree for PH rats relative to $\mathrm{SH}$ rats within each nicotine dose. In addition, delay discounting was significantly reduced at a lower dose for PH F344 relative to SH F344 rats. This suggests that delay discounting of PH rats was more sensitive to nicotine’s effects. Floor effects observed at baseline for SH rats may have contributed to this effect. However, it seems unlikely that results obtained during acute nicotine administration were due to this difference, given that nicotine reduced delay discounting (i.e., reduced impulsive choice) regardless of housing condition and rat strain. Perry et al. (2008) found that methylphenidate reduced delay discounting when choice was more impulsive but had no effect when choice was more self-controlled. Given their results, it seems as though nicotine (i.e., a stimulant-like drug) would have had a more profound effect on delay discounting approaching floor. Thus, it is unclear why nicotine reduced delay discounting to a greater degree in $\mathrm{PH}$ rats relative to $\mathrm{SH}$ rats in the present study.

Furthermore, because delay discounting for PH rats stabilized on variable delay series, while all SH rats responded on a 40-s delay series, delay series are somewhat confounded. In addition, functional equivalence (examined via AUC) was not found between PH LEW rats responding on 16-s and 40-s terminal delay series during baseline. AUC for responding on the 40-s delay series was significantly larger relative to responding on the 16-s series, and delaydiscounting functions for PH LEW rats responding on the 40-s series were flatter compared to all other curves. This flatness may be an indication of behavioral perseveration. Reducing the frequency of 0-s probe sessions in the present study may have contributed to this effect. 
However, because forced-exposure trials were incorporated at the start of each block of trials, it seems unlikely that behavior would perseverate. In addition, within-block choices were examined (data not shown) and responding during free-choice trials did not depend on responding during forced-exposure trials. Thus, it is unlikely that behavioral perseveration occurred in the present study.

Finally, given that data from $\mathrm{PH}$ rats were compared to archival data from SH rats (Anderson \& Diller, 2010), experiments were conducted at different times. Therefore, different experimenters conducted all outlined procedures between the two studies, including handling and performing injections. This could have influenced the obtained results. However, rats were age and weight-matched at the start of the current experiment to help combat this limitation. Future research could be designed to evaluate equivalent groups of SH and PH LEW and F344 rats simultaneously to determine whether results are similar as those obtained with using archival data. Despite these limitations, it seems as though paired housing had a significant effect on delay discounting in LEW and F344 rats, and that previously reported effects of environmental enrichment (Kirkpatrick et al., 2013; 2014; Perry et al., 2008) are at least, in part, due to social housing. Furthermore, the present study suggests several possibilities for future research on neural and environmental determinants of impulsive choice. 


\section{References}

American Psychiatric Association. (2013). Diagnostic and statistical manual of mental disorders: DSM-5. Washington, D.C.: American Psychiatric Association.

Anderson, K.G., \& Woolverton, W.L. (2005). Effects of clomipramine on self-control choice in Lewis and Fischer 344 rats. Pharmacology Biochemistry and Behavior, 80(3), 387-393. doi: 10.1016/j.pbb.2004.11.015

Anderson, K.G., \& Diller, J.W. (2010). Effects of acute and repeated nicotine administration on delay discounting in Lewis and Fischer 344 rats. Behavioural Pharmacology, 21, 754764. doi: 10.1097/FBP.0b013e328340e050

Aparicio, C.F., Elcoro, M., \& Alonso-Alvarez, B. (2015). A long-term study of the impulsive choices of Lewis and Fischer 344 rats. Learning and Behavior, 43, 251-271. doi: 10.3758/s13420-015-0177-y

Benowitz, N.L. (2009). Pharmacology of nicotine: Addiction, smoking-induced disease, and therapeutics. Annual Review of Pharmacology and Toxicology, 49, 57-71. doi: 10.1146/annurev.pharmtox.48.113006.094742

Bickel, W.K., Odum, A.L., \& Madden, G.J. (1999). Impulsivity and cigarette smoking: delay discounting in current, never, and ex-smokers. Psychopharmacology, 146(4), 447-454.

Cadoni, C., Muto, T., \& Di Chiara, G. (2009). Nicotine differentially affects dopamine transmission in the nucleus accumbens shell and core of Lewis and Fischer 344 rats. Neuropharmacology, 57(5-6), 496-501. doi: 10.1016/j.neuropharm.2009.07.033

Dallery, J., \& Locey, M.L. (2005). Effects of acute and chronic nicotine on impulsive choice in rats. Behavioral Pharmacology, 16(1), 15-23. 
Evenden, J.L., \& Ryan, C.N. (1996). The pharmacology of impulsive behavior in rats: the effects of drugs on response choice with varying delays of reinforcement. Psychopharmacology, $128,161-170$.

Fields, S., Collins, C., Leraas, K., Reynolds, B. (2009). Dimensions of impulsive behavior in adolescent smokers and nonsmokers. Experimental and Clinical Psychopharmacology, 17(5), 302-311. doi: 10.1037/a0017185

Flores, G., Wood, G.K., Barbeau, D., Quirion, R., \& Srivastava, L.K. (1998). Lewis and Fischer rats: a comparison of dopamine transporter and receptors levels. Brain Research, 814(12), 34-40. doi: 10.1016/S0006-8993(98)01011-7

Garrido, P., De Blas, M., Ronzoni, G., . .., \& Mora, F. (2013). Differential effects of environmental enrichment and isolation housing on the hormonal and neurochemical responses to stress in the prefrontal cortex of the adult rat: relationship to working and emotional memories. Journal of Neural Transmission, 120, 829-843.

Green, T.A., Cain, M.E., Thompson, M., \& Bardo, M.T. (2003). Environmental enrichment decreases nicotine-induced hyperactivity in rats. Psychopharmacology, 170, 235-241. doi: 10.1007/s00213-003-1538-3

Heyman, G.M., \& Gibb, S.P. (2006). Delay discounting in college cigarette chippers. Behavioral Pharmacology, 17(8), 669-679.

Holt, D.D., Green, L., \& Myerson, J. (2012). Estimating the subjective value of future rewards: comparison of adjusting-amount and adjusting-delay procedures. Behavioural Processes, 90(3), 302-310. doi: 10.1016/j.beproc.2012.03.003 
Huskinson, S.L., \& Anderson, K.G. (2012). Effects of acute and chronic administration of diazepam on delay discounting in Lewis and Fischer 344 rats. Behavioural Pharmacology, 23, 315-330. doi: 10.1097/FBP.0b013e3283564da4

Huskinson, S.L., Krebs, C.A., \& Anderson, K.G. (2012). Strain differences in delay discounting between Lewis and Fischer 344 rats at baseline and following acute and chronic administration of $d$-amphetamine. Pharmacology, Biochemistry, and Behavior, 101, 403416. doi: 10.1016/j.pbb.2012.02.005

Jones, G.H., Hernandez, T.D., Kendall, D.A., Marsden, C.A., \& Robbins, T.W. (1992). Dopaminergic and serotonergic function following isolation rearing in rats: Study of behavioural responses and postmortem and in vivo neurochemistry. Pharmacology, Biochemistry, and Behavior, 43, 17-35.

Kayir, H., Semenova, S., \& Markou, A. (2014). Baseline impulsive choice predicts the effects of nicotine and nicotine withdrawal on impulsivity in rats. Progress in NeuroPsychopharmacology \& Biological Psychiatry, 48, 6-13. doi: 10.1016/j.pnpbp.2013.09.007

Kirkpatrick, K., Marshall, A.T., Clarke, J., \& Cain, M.R. (2013). Environmental rearing effects on impulsivity and reward sensitivity. Behavioral Neuroscience, 127(5), 712-724. doi: 10.1037/a0034124

Kirkpatrick, K., Marshall, A.T., Smith, A.P., Koci, J., \& Park, Y. (2014). Individual differences in impulsive and risky choice: Effects of environmental rearing conditions. Behavioural Brain Research, 269, 115-127. 
Locey, M.L., \& Dallery, J. (2009). Isolating behavioral mechanisms of intertemporal choice: nicotine effects on delay discounting and amount sensitivity. Journal of the Experimental Analysis of Behavior, 91(2), 213-223.

Madden, G.J., Smith, N.G., Brewer, A.T., Pinkston, J.W., \& Johnson, P.S. (2008). Steady-state assessment of impulsive choice in Lewis and Fischer F344 rats: Between-condition delay manipulations. Journal of the Experimental Analysis of Behavior, 90(3), 333-344.

Mazur, J.E. (1987). An adjusting procedure for studying delayed reinforcement. Quantitative Analyses of Behavior, 5, 55-73.

Mendez, I.A., Gilbert, R.J., Bizon, J.L., \& Setlow, B. (2012) Effects of acute administration of nicotinic and muscarinic cholinergic agonists and antagonists on performance in different cost-benefit decision-making tasks in rats. Psychopharmacology, 224(4), 489-499. doi: 10.1007/s00213-012-2777-y

Miura, H., Qiao, H., \& Ohta, T. (2002). Attenuating effects of the isolated rearing condition on increased brain serotonin and dopamine turnover elicited by novelty stress. Brain Research, 926, 10-17.

Mobini, S., Chiang, T.J., Al-Ruwaitea, A.S., Ho, M.Y., Bradshaw, C.M., \& Szabadi, E. (2000). Effect of central 5-hydroxytryptamine depletion on inter-temporal choice: a quantitative analysis. Psychopharmacology, 149(3), 313-318.

Myerson, J., Green, L., \& Warusawitharana, M. (2001). Area under the curve as a measure of discounting. Journal of the Experimental Analysis of Behavior, 76, 235-243.

Perry, J.L., Stairs, D.J., \& Bardo, M.T. (2008). Impulsive choice and environmental enrichment: Effects of d-amphetamine and methylphenidate. Behavioural Brain Research, 193, 4854. doi: 10.1016/j.bbr.2008.04.019 
Richards, J.B., Lloyd, D.R., Kuehlewind, B., Militello, L., Paredez, M., Woods, L.S., \& Palmer, A.A. (2013). Strong genetic influences on measures of behavioral-regulation among inbred rat strains. Genes, Brain, and Behavior, 12, 490-502. doi: 10.1111/gbb.12050

Selim, M., \& Bradberry, C.W. (1996). Effect of ethanol on extracellular 5-HT and glutamate in the nucleus accumbens and prefrontal cortex: comparison between the Lewis and Fischer 344 rat strains. Brain Research, 716(1-2), 157-164. doi: 10.1016/0006-8993(95)01385-7

Stein, J.S., Pinkston, J.W., Brewer, A.T., Francisco, M.T., \& Madden, G.J. (2012). Delay discounting in Lewis an d Fischer F344 rats: Steady-state and rapid-determination adjusting-amount procedures. Journal of the Experimental Analysis of Behavior, 97(3), 305-321.

Stairs, D.J., \& Bardo, M.T. (2009). Neurobehavioral effects of environmental enrichment and drug abuse vulnerability. Pharmacology Biochemistry and Behavior, 92, 377-382. doi: 10.1016/j.pbb.2009.01.016

Summers, K.L., \& Giacobini, E. (1995). Effects of local and repeated systemic administration of (-)nicotine on extracellular levels of acetylcholine, norepinephrine, dopamine, and serotonin in rat cortex. Neurochemical Research, 20(6), 753-759.

Sziraki, I., Lipovac, M.N., Hashim, A., . ., \& Lajtha, A. (2001). Differences in nicotine-induced dopamine release and nicotine pharmacokinetics between Lewis and Fischer 344 rats. Neurochemical Research, 26, 609-617. doi: 10.1023/A:1010979018217

Wilhelm, C.J., \& Mitchell, S.H. (2009). Strain differences in delay discounting using inbred rats. Genes, Brain, and Behavior, 8(4), 426-434. doi: 10.1111/j.1601-183X.2009.00484.x 
Winstanley, C.A., Dalley, J.W., Theobald, D.E., \& Robbins, T.W. (2003). Global 5-HT depletion attenuates the ability of amphetamine to decrease impulsive choice on delay-discounting task in rats. Psychopharmacology, 170(3), 320-331.

Winstanley, C.A., Theobald, D.E., Dalley, J.W., Cardinal, R.N., \& Robbins, T.W. (2006). Double dissociation between serotonergic and dopaminergic modulation of medial prefrontal and orbitofrontal cortex during a test of impulsive choice. Cerebral Cortex, 16, 106-114. doi: 10.1093/cercor/bhi088

Zeeb, F.D., Soko, A.D., Ji, X., \& Fletcher, P.J. (2016). Low impulsive action, but not impulsive choice, predicts greater conditioned reinforcer salience and augmented nucleus accumbens dopamine release. Neuropsychopharmacology. [Epub ahead of print]. doi: 10.1038/npp.2016.9

Zhu, J., Bardo, M.T., \& Dwoskin, L.P. (2013). Distinct effects of enriched environment on dopamine clearance in nucleus accumbens shell and core following systemic nicotine administration. Synapse, 67, 57-67. doi: 10.1002/syn.21615

Zhu, J., Bardo, M.T., Green, T.A., Wedlund, P.J., \& Dwoskin, L.P. (2007). Nicotine increases dopamine clearance in medial prefrontal cortex in rats raised in an enriched environment. Journal of Neurochemistry, 103, 2575-2588. doi: 10.1111/j.1471-4159.2007.04951.x 
Table 1. Statistical analyses for all outcome measures.

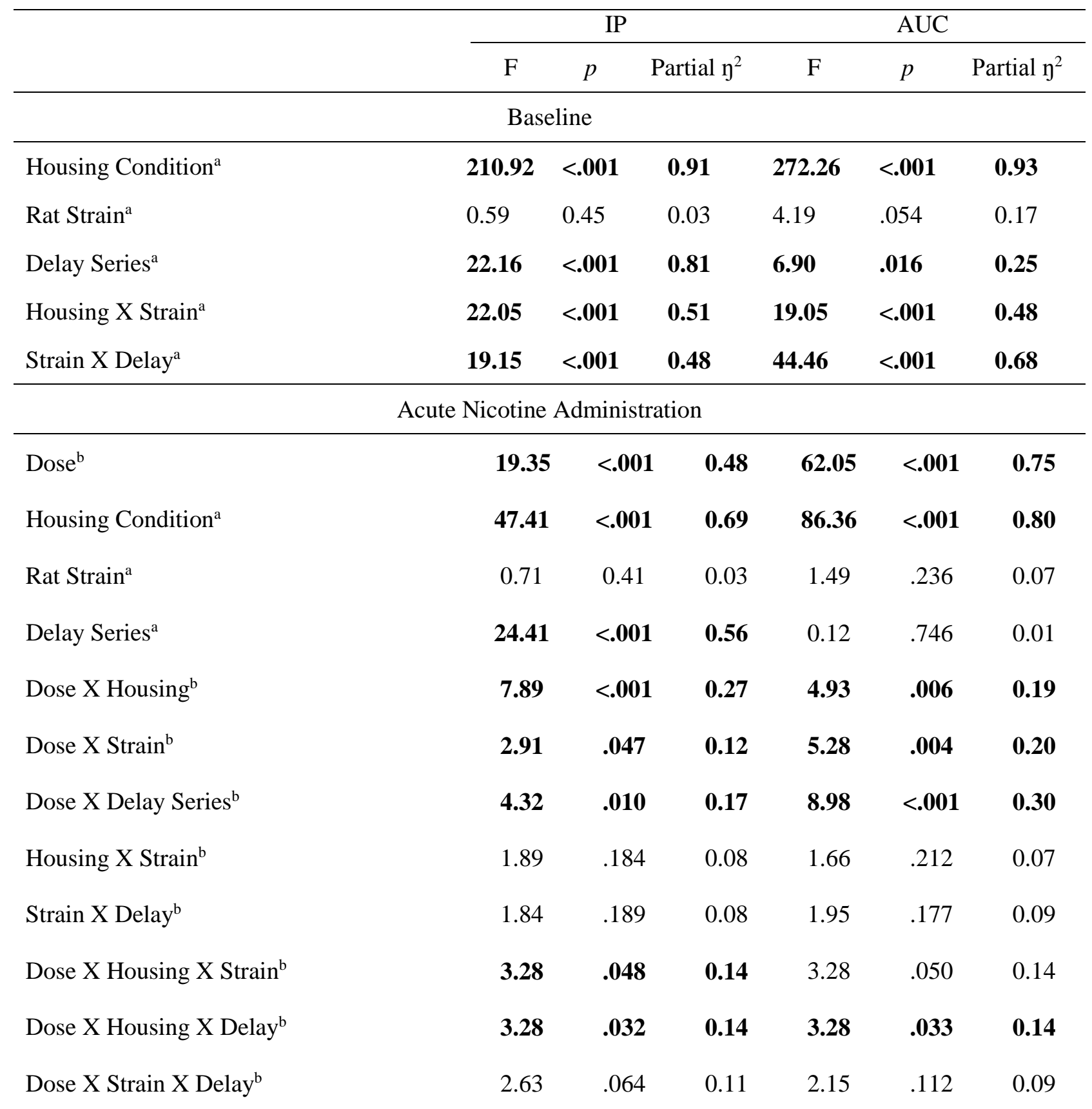

Note: Bolded values denote statistical significance, $p<.05$.

${ }^{\mathrm{a}} d f=(1,21)$

${ }^{\mathrm{b}} d f=(3,63)$ 
Table 2.

Percent larger-reinforcer choice across blocks, indifference points (IP), and area under the curve (AUC) at baseline.

\begin{tabular}{|c|c|c|c|c|c|c|c|}
\hline \multirow{2}{*}{$\begin{array}{l}\text { Subject ID } \\
\text { Pair-housed }\end{array}$} & \multicolumn{5}{|c|}{ \% Larger-Reinforcer Choice } & \multirow[t]{2}{*}{ IP (s) } & \multirow[t]{2}{*}{ AUC } \\
\hline & Block: 1 & 2 & 3 & 4 & 5 & & \\
\hline JOL1 (40s) & 93.8 & 84.8 & 45.4 & 27.4 & 16.8 & 15.2 & 0.43 \\
\hline JOL2 (40s) & 88.1 & 63.4 & 38.4 & 23.3 & 29.9 & 13.1 & 0.40 \\
\hline JOL3 (16s) & 94.3 & 72.2 & 18.8 & 7.4 & 0.0 & 2.9 & 0.20 \\
\hline JOL4 (40s) & 93.6 & 79.1 & 58.3 & 37.4 & 21.0 & 10.4 & 0.53 \\
\hline JOL5 (16s) & 100.0 & 86.1 & 34.3 & 11.2 & 5.76 & 4.2 & 0.30 \\
\hline JOL6 (40s) & 94.6 & 65.8 & 28.1 & 26.4 & 42.1 & 17.6 & 0.45 \\
\hline Mean & 94.1 & 75.2 & 37.2 & 22.2 & 19.2 & 10.6 & 0.38 \\
\hline SEM & 1.54 & 3.93 & 5.61 & 4.53 & 6.33 & 2.44 & 0.05 \\
\hline JOF1 (16s) & 100.0 & 92.4 & 57.3 & 14.9 & 5.7 & 5.61 & 0.38 \\
\hline JOF2 (40s) & 100.0 & 97.0 & 53.0 & 3.1 & 4.6 & 9.27 & 0.34 \\
\hline JOF3 (16s) & 98.8 & 98.8 & 49.9 & 3.6 & 1.2 & 4.69 & 0.36 \\
\hline JOF4 (16s) & 100.0 & 91.6 & 57.0 & 20.9 & 5.6 & 5.99 & 0.44 \\
\hline JOF5 (16s) & 100.0 & 98.7 & 50.2 & 10.3 & 6.5 & 6.68 & 0.48 \\
\hline JOF6 (40s) & 98.7 & 93.5 & 52.7 & 10.4 & 2.6 & 9.93 & 0.31 \\
\hline Mean & 99.6 & 95.3 & 53.3 & 10.5 & 4.4 & 7.03 & 0.39 \\
\hline SEM & 0.27 & 1.32 & 1.32 & 2.77 & 0.83 & 0.86 & 0.03 \\
\hline \multicolumn{8}{|c|}{ Singly housed (40s) } \\
\hline L30-1 & 91.6 & 21.6 & 2.5 & 0.0 & 3.6 & 3.24 & 0.10 \\
\hline L30-2 & 89.7 & 18.1 & 5.7 & 2.8 & 2.8 & 2.95 & 0.11 \\
\hline L30-3 & 91.6 & 24.9 & 4.2 & 2.8 & 0.0 & 3.41 & 0.11 \\
\hline L30-4 & 92.3 & 22.3 & 4.2 & 2.8 & 1.4 & 3.32 & 0.11 \\
\hline L30-5 & 86.3 & 9.3 & 1.6 & 1.6 & 0.0 & 2.23 & 0.07 \\
\hline L30-6 & 90.0 & 15.7 & 3.3 & 1.1 & 0.0 & 2.84 & 0.09 \\
\hline L30-7 & 95.1 & 21.4 & 9.5 & 1.3 & 0.1 & 3.45 & 0.11 \\
\hline L30-8 & 80.0 & 23.9 & 8.4 & 4.9 & 9.6 & 2.77 & 0.14 \\
\hline Mean & 89.6 & 19.6 & 4.9 & 2.2 & 2.2 & 3.03 & 0.10 \\
\hline SEM & 1.63 & 1.82 & 0.99 & 0.52 & 1.17 & 0.15 & 0.01 \\
\hline F30-1 & 95.2 & 46.3 & 10.9 & 2.4 & 2.4 & 4.86 & 0.15 \\
\hline F30-2 & 97.9 & 42.8 & 4.2 & 2.1 & 1.1 & 4.62 & 0.13 \\
\hline F30-3 & 93.8 & 36.4 & 11.6 & 5.3 & 1.1 & 4.16 & 0.15 \\
\hline F30-5 & 92.2 & 39.6 & 1.1 & 0.0 & 2.1 & 4.27 & 0.11 \\
\hline F30-6 & 96.4 & 36.9 & 7.1 & 0.0 & 2.4 & 4.25 & 0.13 \\
\hline F30-7 & 95.5 & 36.7 & 5.6 & 2.3 & 1.1 & 4.21 & 0.13 \\
\hline F30-8 & 92.8 & 48.7 & 10.8 & 2.4 & 4.9 & 4.99 & 0.16 \\
\hline Mean & 94.8 & 41.1 & 7.3 & 2.1 & 2.2 & 4.48 & 0.14 \\
\hline SEM & 0.77 & 1.88 & 1.50 & 0.67 & 0.51 & 0.13 & 0.01 \\
\hline
\end{tabular}

Note: The number in parenthesis represents individual-subject delay series. 
Table 3.

Indifference points (IP) and area under the curve (AUC) across doses of acute nicotine.

\begin{tabular}{|c|c|c|c|c|c|c|c|c|c|c|}
\hline \multirow{2}{*}{$\begin{array}{l}\text { Subject ID } \\
\text { Pair-housed }\end{array}$} & \multicolumn{2}{|c|}{ Control } & \multicolumn{2}{|c|}{ Saline } & \multicolumn{2}{|c|}{$1.0 \mathrm{mg} / \mathrm{kg}$} & \multicolumn{2}{|c|}{$0.3 \mathrm{mg} / \mathrm{kg}$} & \multicolumn{2}{|c|}{$0.1 \mathrm{mg} / \mathrm{kg}$} \\
\hline & $\mathrm{IP}(\mathrm{s})$ & AUC & $\mathrm{IP}(\mathrm{s})$ & AUC & $\mathrm{IP}(\mathrm{s})$ & AUC & $\mathrm{IP}(\mathrm{s})$ & AUC & $\mathrm{IP}(\mathrm{s})$ & AUC \\
\hline JOL1 (16s) & 4.89 & 0.39 & 3.60 & 0.32 & 9.09 & 0.78 & 4.96 & 0.42 & 4.94 & 0.37 \\
\hline JOL2 (40s) & 10.05 & 0.37 & 6.52 & 0.29 & 12.69 & 0.40 & 8.00 & 0.31 & 8.71 & 0.37 \\
\hline JOL3 (16s) & 2.78 & 0.21 & 3.14 & 0.23 & 11.42 & 0.61 & 5.95 & 0.43 & 3.70 & 0.25 \\
\hline JOL4 (40s) & 10.01 & 0.46 & 8.60 & 0.35 & 14.58 & 0.56 & 26.36 & 0.53 & 16.61 & 0.46 \\
\hline JOL5 (16s) & 3.48 & 0.29 & 3.40 & 0.28 & 6.18 & 0.42 & 5.54 & 0.37 & 3.65 & 0.29 \\
\hline JOL6 (40s) & 8.40 & 0.40 & 17.88 & 0.46 & 33.52 & 0.62 & 20.26 & 0.48 & 31.73 & 0.52 \\
\hline Mean & 6.60 & 0.35 & 7.19 & 0.32 & 14.58 & 0.56 & 11.84 & 0.42 & 11.56 & 0.38 \\
\hline SEM & 1.34 & 0.04 & 2.31 & 0.03 & 4.35 & 0.06 & 4.09 & 0.03 & 4.50 & 0.04 \\
\hline JOF1 (16s) & 4.75 & 0.36 & 2.88 & 0.19 & 7.99 & 0.58 & 6.65 & 0.44 & 3.70 & 0.28 \\
\hline JOF2 (16s) & 4.08 & 0.31 & 3.81 & 0.31 & 17.25 & 0.78 & 6.75 & 0.47 & 3.42 & 0.28 \\
\hline JOF3 (16s) & 4.00 & 0.30 & 4.82 & 0.35 & 9.95 & 0.60 & 5.46 & 0.37 & 3.60 & 0.24 \\
\hline JOF4 (16s) & 5.08 & 0.38 & 4.14 & 0.35 & 19.66 & 0.78 & 6.53 & 0.45 & 6.41 & 0.42 \\
\hline JOF5 (16s) & 4.01 & 0.33 & 4.18 & 0.31 & 15.84 & 0.83 & 7.03 & 0.49 & 5.16 & 0.37 \\
\hline JOF6 (40s) & 10.51 & 0.32 & 7.33 & 0.23 & 15.79 & 0.47 & 21.07 & 0.53 & 10.01 & 0.33 \\
\hline Mean & 5.40 & 0.33 & 4.53 & 0.29 & 14.41 & 0.67 & 8.92 & 0.46 & 5.38 & 0.32 \\
\hline SEM & 1.04 & 0.01 & 0.62 & 0.03 & 1.83 & 0.06 & 2.44 & 0.02 & 1.04 & 0.03 \\
\hline \multicolumn{11}{|c|}{ Singly housed (40s) } \\
\hline L30-1 & 4.06 & 0.11 & 4.05 & 0.11 & 4.49 & 0.15 & 4.81 & 0.12 & 2.94 & 0.09 \\
\hline L30-2 & 4.00 & 0.15 & 5.24 & 0.18 & 4.09 & 0.15 & 4.58 & 0.18 & 4.73 & 0.15 \\
\hline L30-3 & 4.34 & 0.16 & 5.75 & 0.18 & 5.62 & 0.19 & 6.17 & 0.17 & 3.10 & 0.17 \\
\hline L30-4 & 3.50 & 0.08 & 3.42 & 0.13 & 4.30 & 0.12 & 4.58 & 0.13 & 2.50 & 0.07 \\
\hline L30-5 & 2.36 & 0.07 & 3.26 & 0.10 & 4.05 & 0.12 & 3.95 & 0.08 & 3.46 & 0.09 \\
\hline L30-6 & 3.13 & 0.09 & 4.14 & 0.09 & 3.87 & 0.12 & 5.17 & 0.14 & 3.64 & 0.11 \\
\hline L30-7 & 3.76 & 0.10 & 3.98 & 0.13 & 4.04 & 0.12 & 5.54 & 0.16 & 7.49 & 0.19 \\
\hline L30-8 & 3.44 & 0.13 & 3.13 & 0.15 & 4.28 & 0.17 & 4.99 & 0.16 & 1.61 & 0.07 \\
\hline Mean & 3.57 & 0.11 & 4.12 & 0.13 & 4.34 & 0.14 & 4.97 & 0.14 & 3.68 & 0.12 \\
\hline SEM & 0.22 & 0.01 & 0.33 & 0.01 & 0.19 & 0.01 & 0.24 & 0.01 & 0.63 & 0.02 \\
\hline F30-1 & 5.47 & 0.15 & 6.57 & 0.19 & 12.98 & 0.41 & 7.37 & 0.22 & 5.83 & 0.15 \\
\hline F30-2 & 5.05 & 0.14 & 5.23 & 0.16 & 11.21 & 0.31 & 8.48 & 0.23 & 5.90 & 0.16 \\
\hline F30-3 & 3.86 & 0.10 & 4.44 & 0.12 & 7.27 & 0.24 & 3.42 & 0.09 & 4.19 & 0.09 \\
\hline F30-5 & 4.35 & 0.14 & 6.08 & 0.18 & 5.01 & 0.18 & 5.30 & 0.14 & 4.50 & 0.10 \\
\hline F30-6 & 3.67 & 0.12 & 3.94 & 0.12 & 4.73 & 0.11 & 3.42 & 0.09 & 2.81 & 0.08 \\
\hline F30-7 & 4.35 & 0.11 & 4.59 & 0.11 & 13.13 & 0.41 & 4.22 & 0.09 & 4.31 & 0.11 \\
\hline F30-8 & 3.96 & 0.13 & 4.22 & 0.16 & 3.70 & 0.15 & 3.86 & 0.15 & 4.02 & 0.10 \\
\hline Mean & 4.39 & 0.13 & 5.01 & 0.15 & 8.29 & 0.26 & 5.15 & 0.14 & 4.51 & 0.11 \\
\hline SEM & 0.25 & 0.01 & 0.37 & 0.01 & 1.54 & 0.05 & 0.76 & 0.02 & 0.41 & 0.01 \\
\hline
\end{tabular}

Note: The number in parenthesis represents individual-subject delay series. 


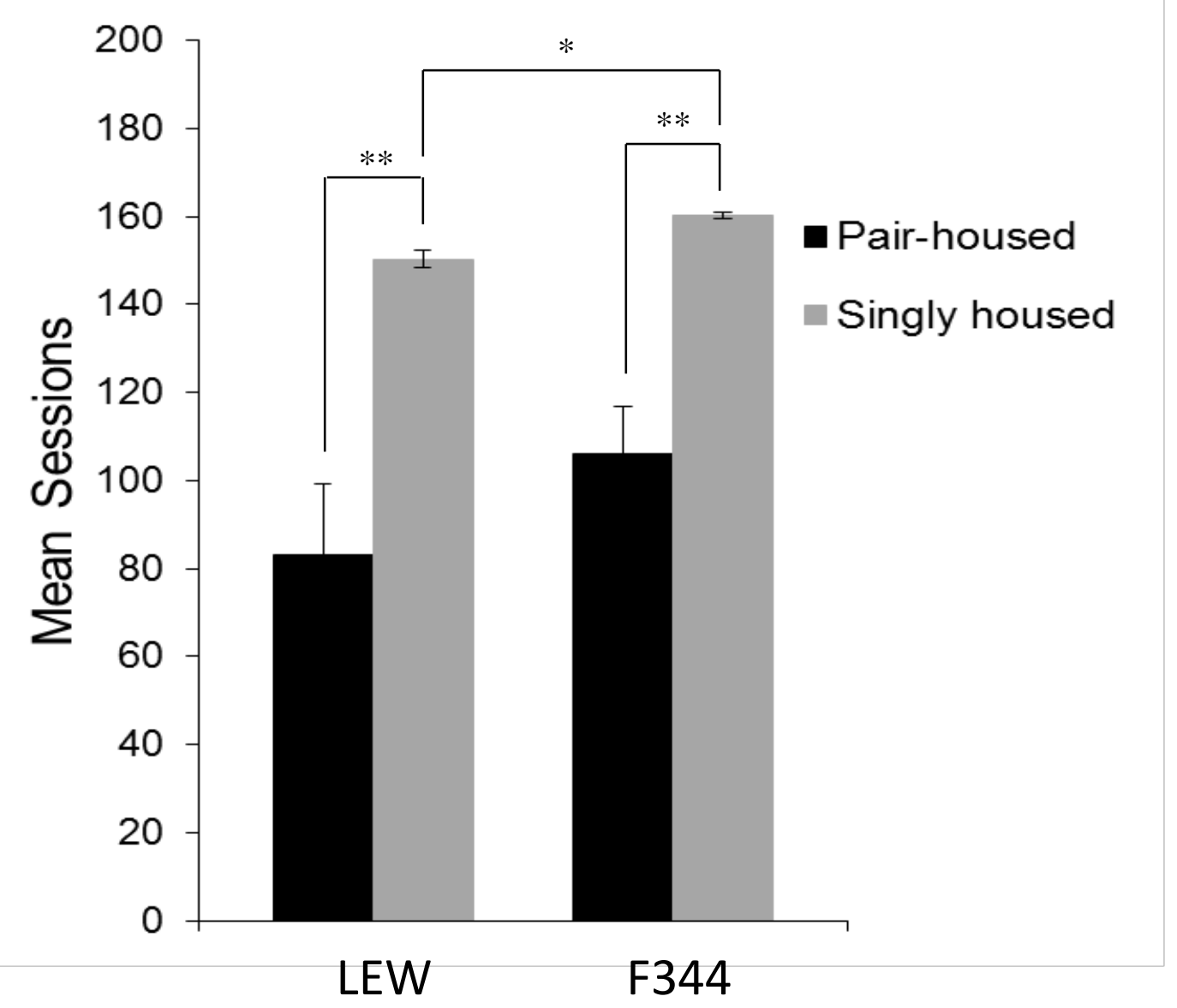

Figure 1. Mean sessions to reach baseline stability for LEW (left) and F344 (right) rats. Error bars represent standard error. Asterisks represent a statistically significant difference between means $(* p<.05 ; * * p<.01)$. 

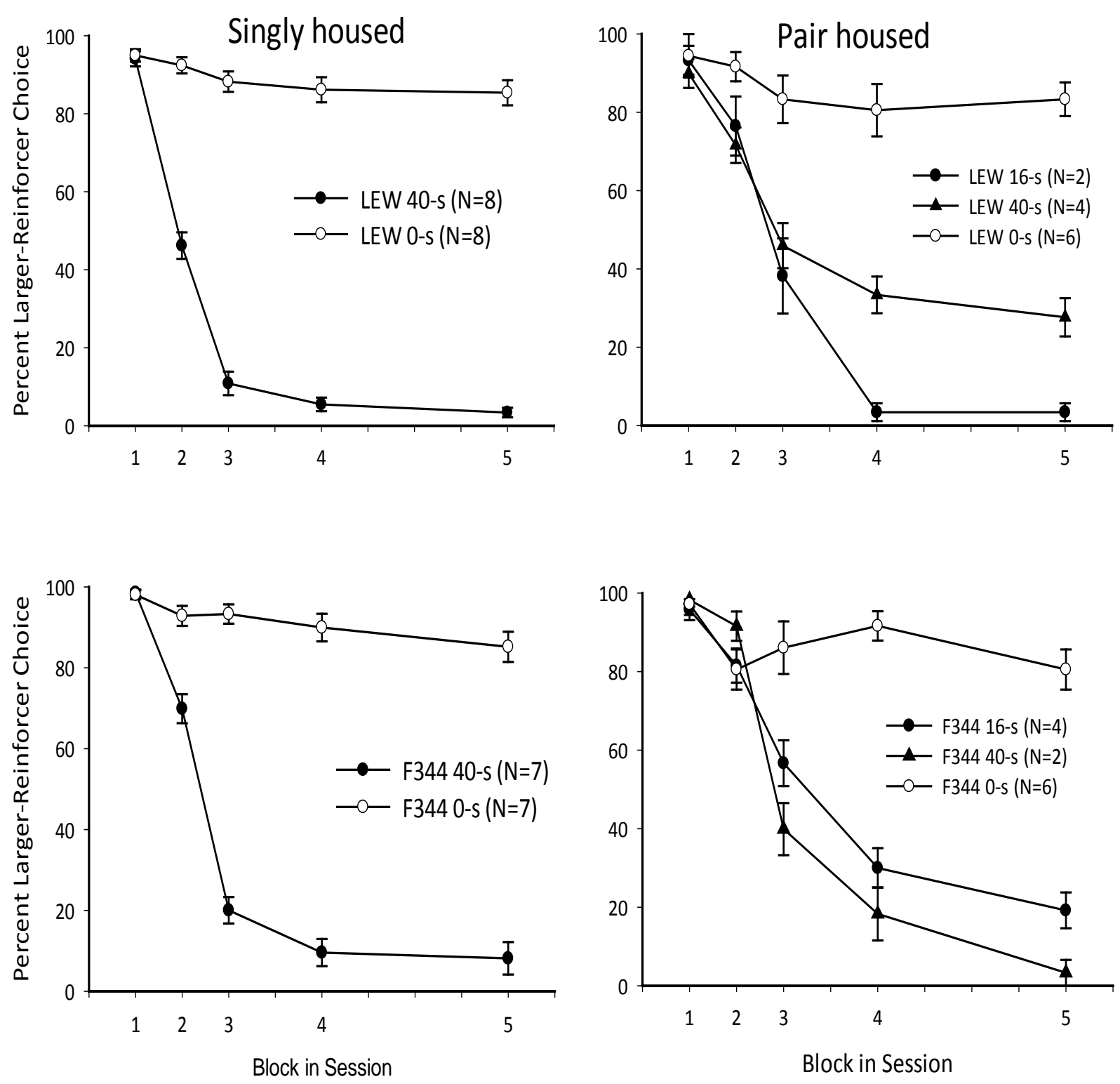

Figure 2. Filled symbols represent delay-discounting curves for singly housed (left panel; Anderson \& Diller, 2010) and pair-housed (right panel) LEW (top) and F344 (bottom) rats. Pairhoused rat data are separated into 16-s and 40-s delay series. Data are presented from the last five sessions of the baseline condition. Unfilled symbols represent mean percent larger-reinforcer choice by LEW and F344 rats during 0-s probe sessions, where all delays are set at 0 s across the session. Error bars represent standard error. 


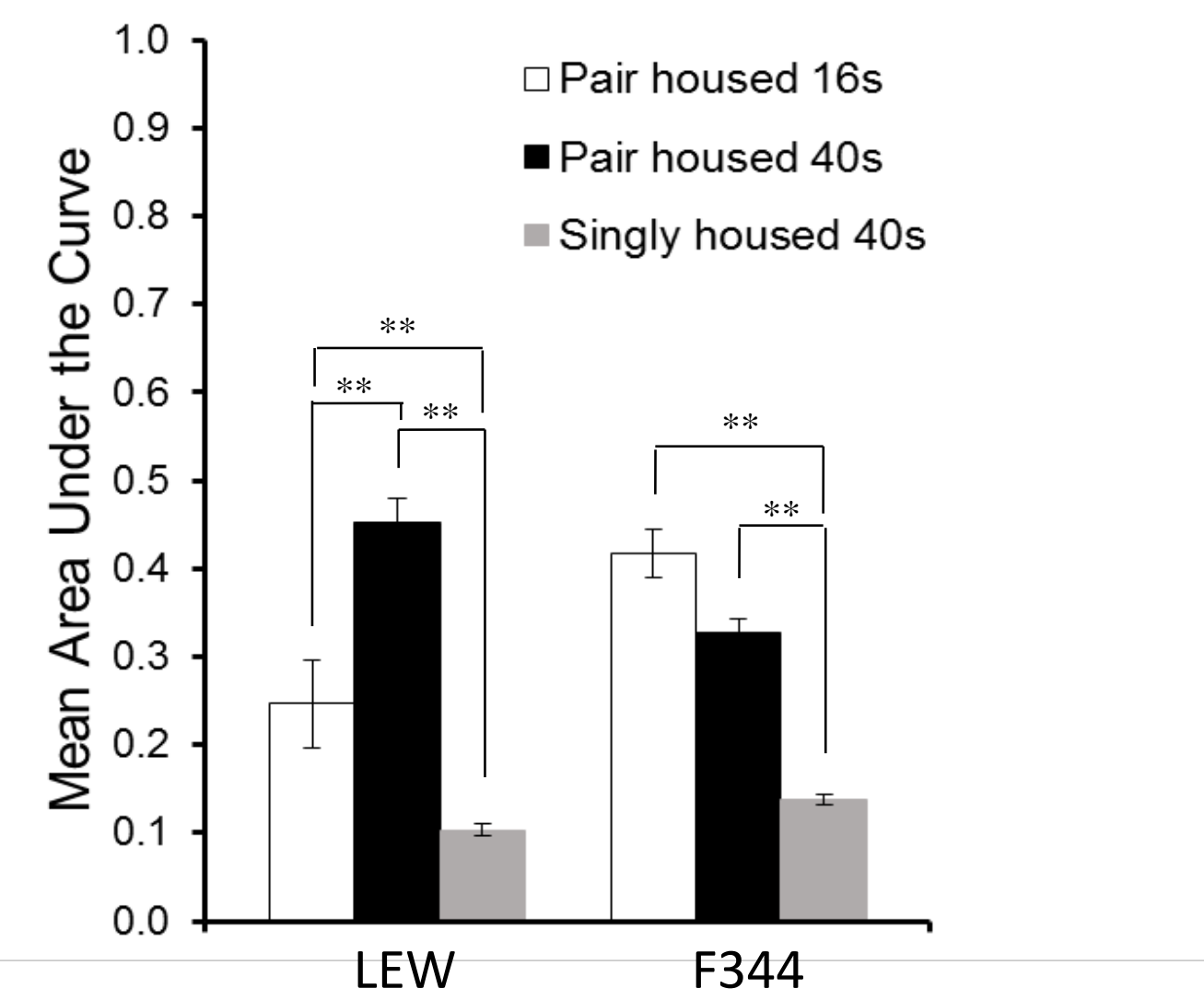

Figure 3. Mean area under the curve (AUC) for LEW (left) and F344 (right) rats. AUC data for PH rats are split into responding on the 16-s (unfilled bar; LEW n=3, F344 n=4) and 40-s (filled black bar; LEW n=3, F344 n=2) delay series. Filled gray bars represent AUC for SH rats (LEW $\mathrm{n}=8$, F344 n=7). AUC ranges from 0.0 (most discounting) to 1.0 (no discounting). Error bars represent standard error. Asterisks represent a statistically significant difference between means $(* * p<.01)$. 


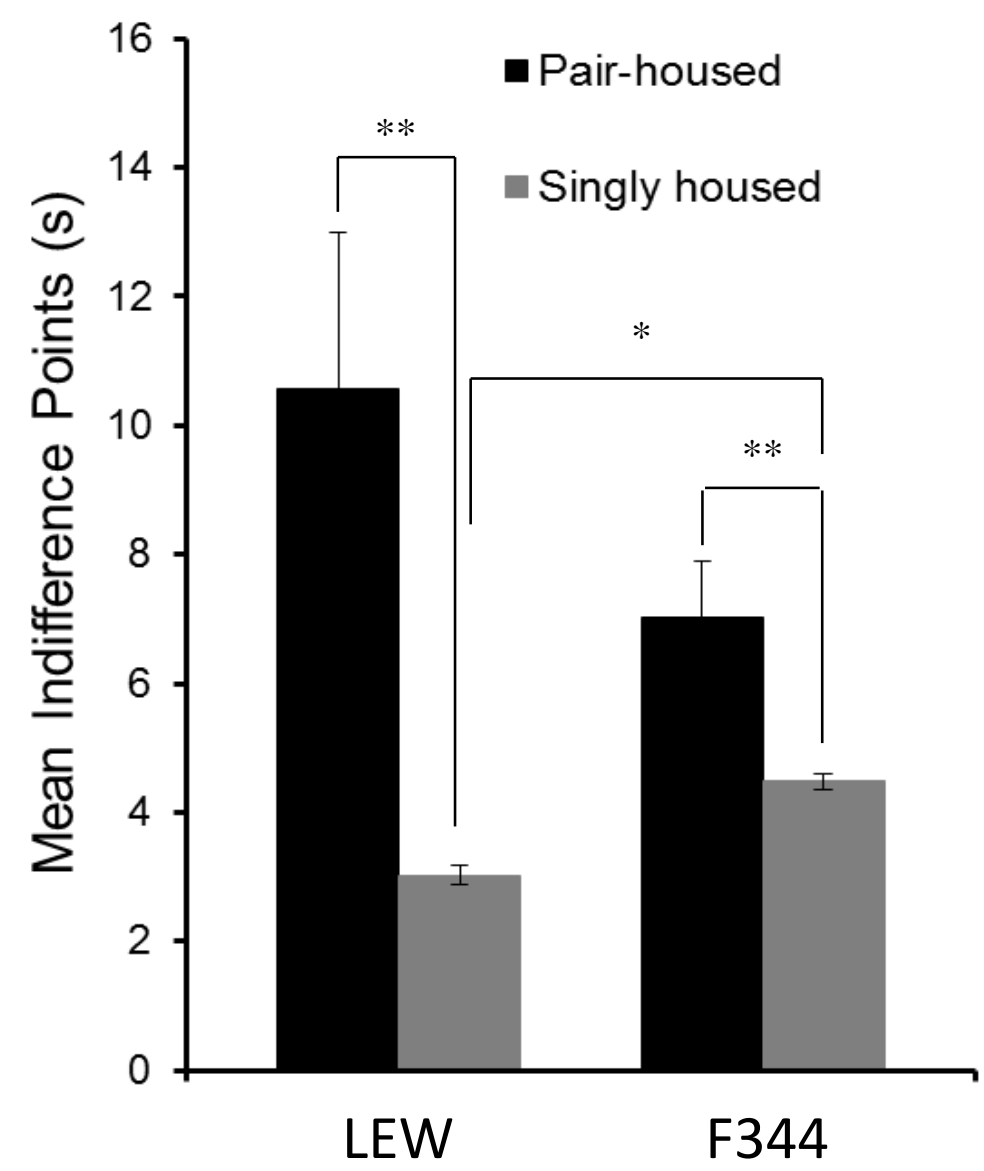

Figure 4. Mean indifference points (s) for LEW (left) and F344 (right) rats. Error bars represent standard error. Asterisks represent a statistically significant difference between means $(* p<.05$; $* * p<.01)$. 

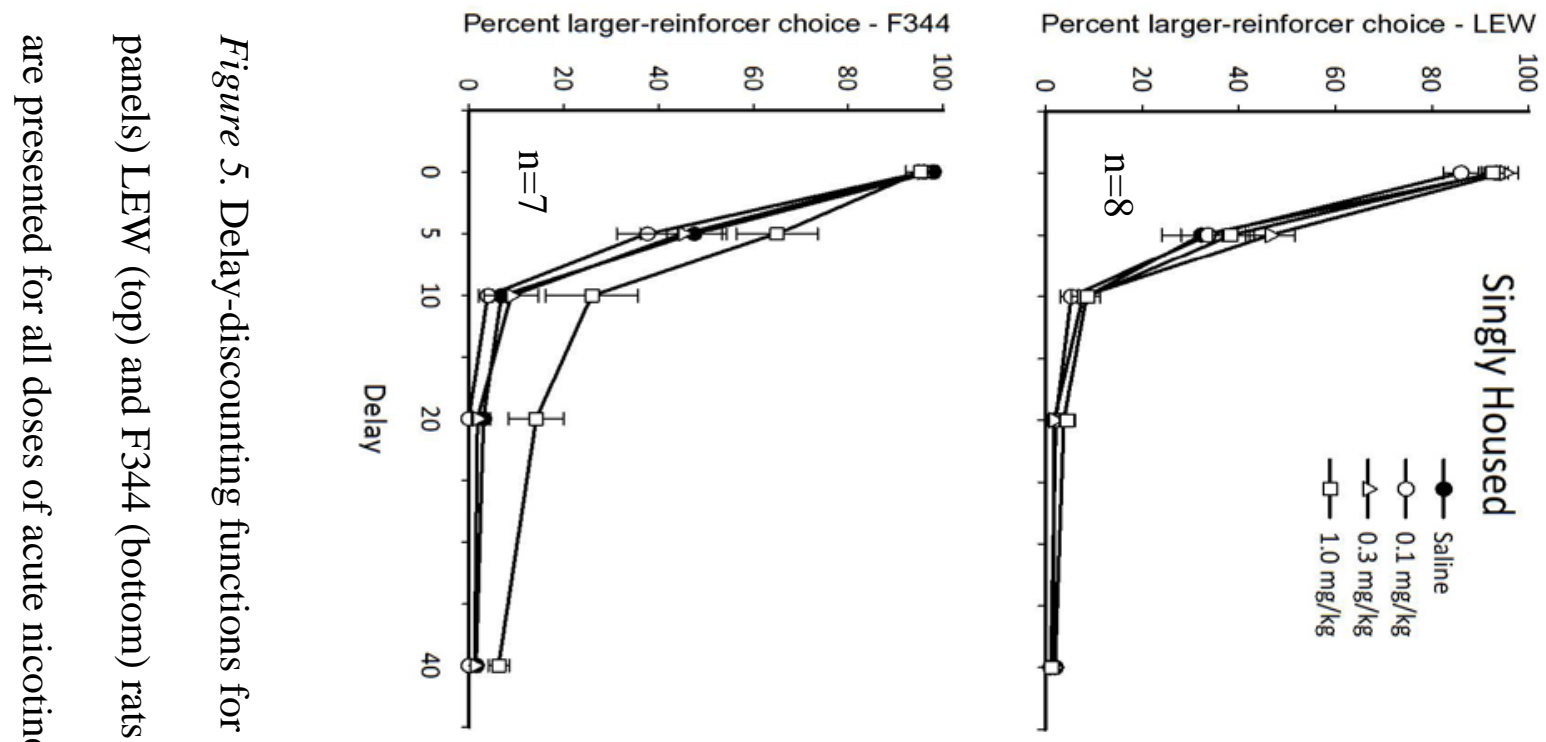

1
0
0
0
0
0
0
0
0
0
0
0
0
0
0
0
0
0
0
0
0
0

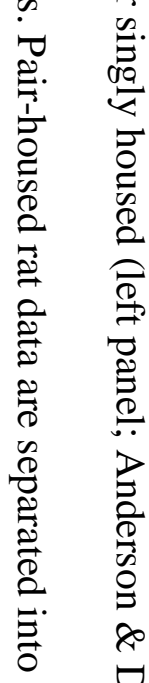

酡
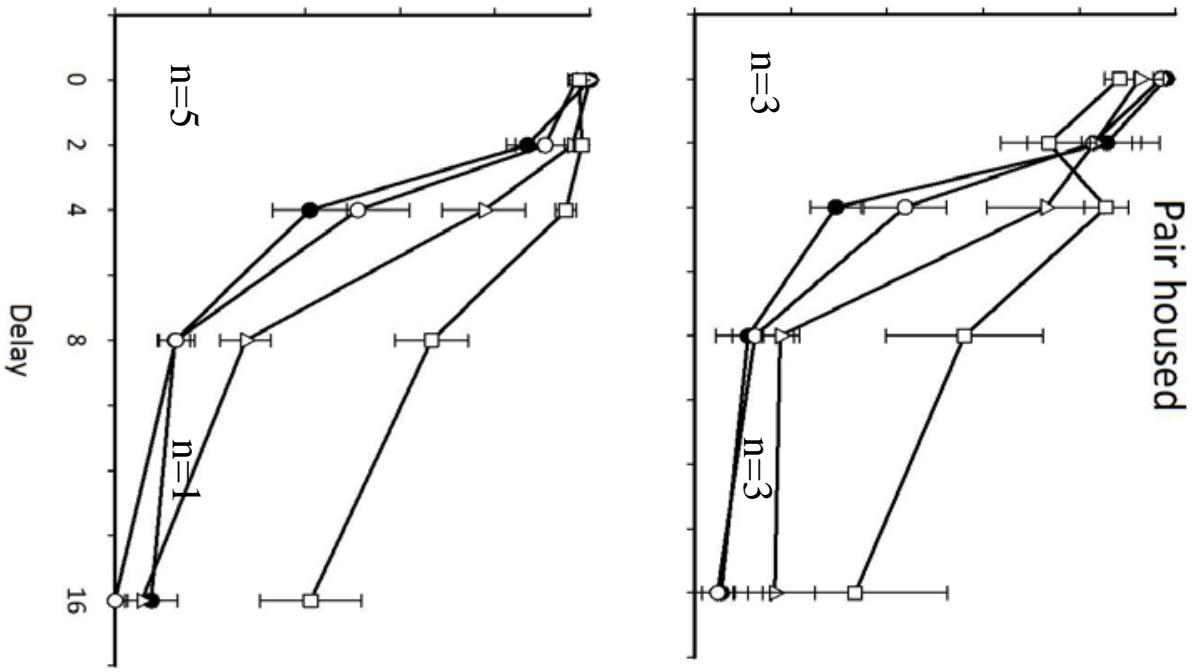

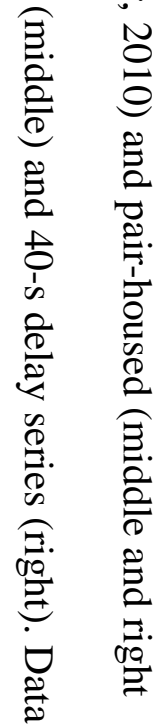
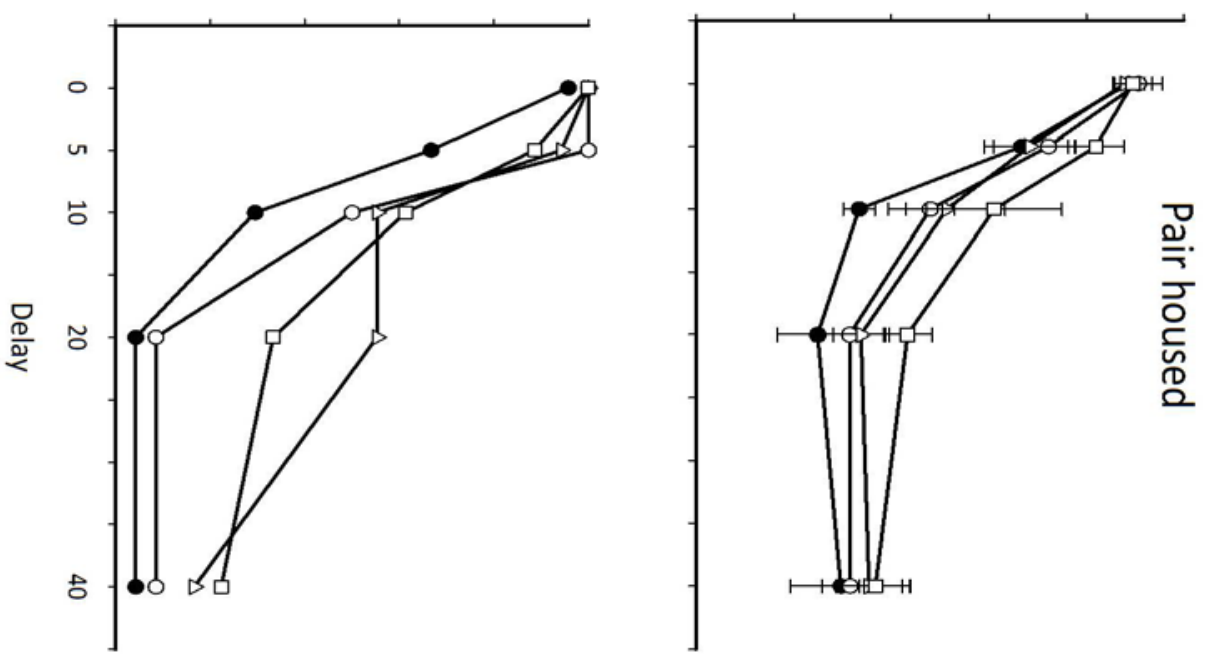

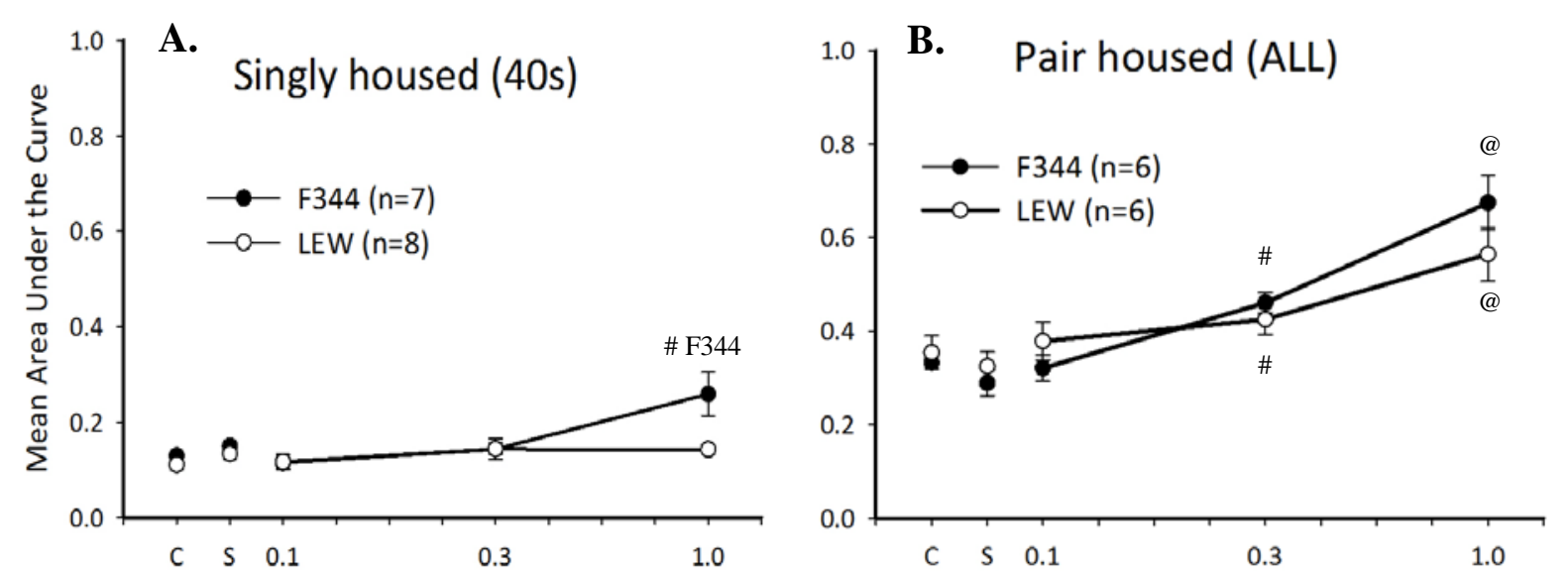

C.
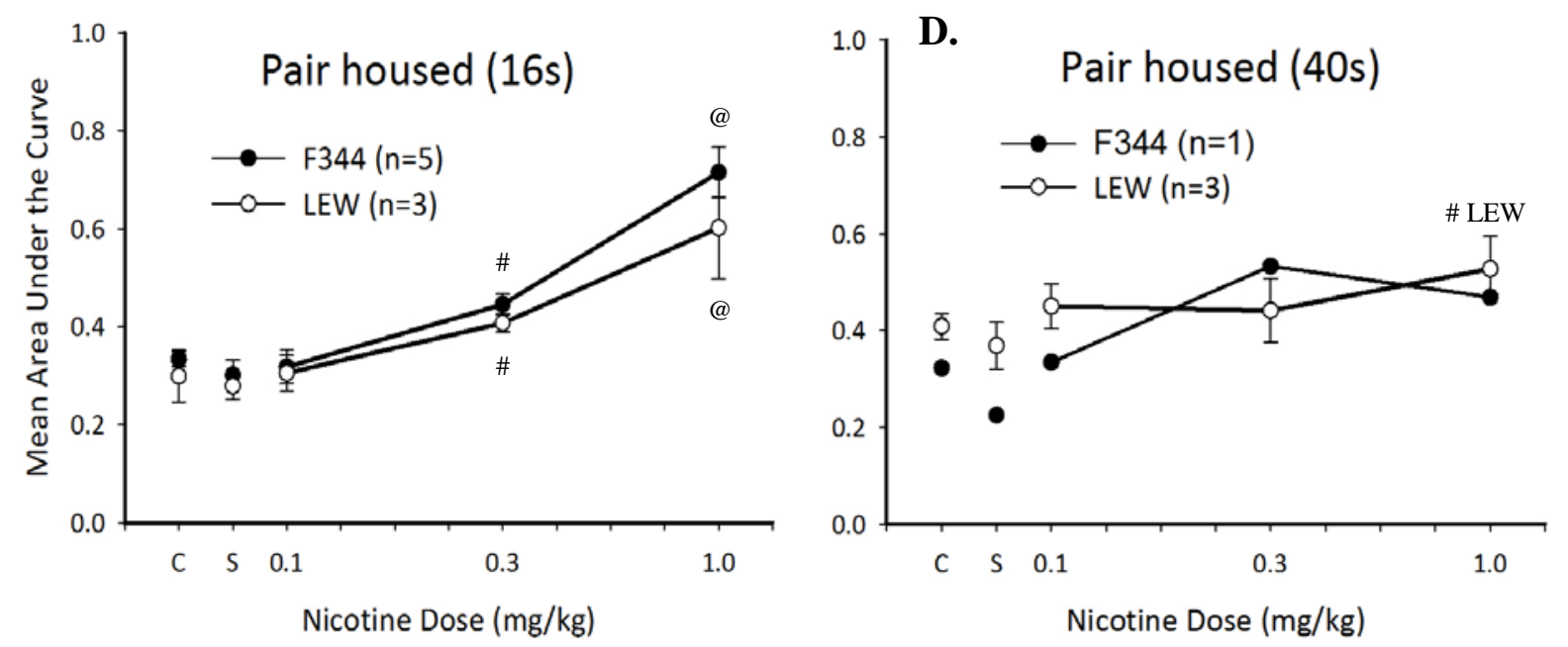

Figure 6. Mean area under the curve (AUC) for singly housed (panel A) and pair-housed (panels B, C, and D) LEW (unfilled circles) and F344 (filled circles) rats. Pair-housed rat data are presented collapsed across delay series (panel B) and separated into 16-s (panel C) and 40-s delay series (panel D). On the x-axis, “C” corresponds to control (non-drug) sessions and "S” corresponds to saline administration. AUC ranges from 0.0 (most discounting) to 1.0 (no discounting). Error bars represent standard error. (\#: $p<.05$, @: $p<.01$ significantly different from saline). 

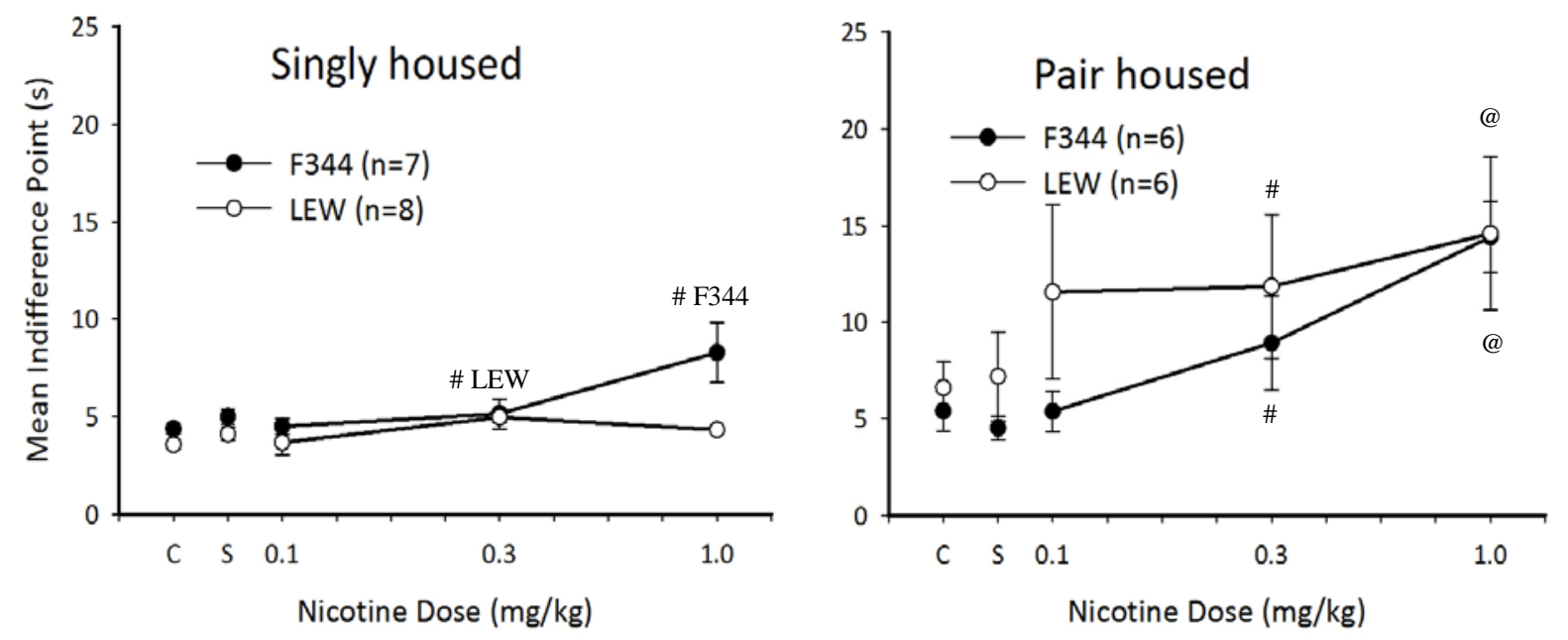

Figure 7. Mean indifference points (s) for singly housed (left) and pair-housed (right) LEW (unfilled circles) and F344 (filled circles) rats across doses of acute nicotine. On the $\mathrm{x}$-axis, "C" corresponded to control (non-drug) sessions and "S" corresponds to saline administration. Error bars represent standard error. (\#: $p<.05$, @: $p<.01$ significantly different from saline). 